\title{
Usages de la ville, usages du corps : Les Promenades de Paris d'Adolphe Alphand
}

Thinking a new town for a new body: Alphand's Promenades de Paris.

\section{Édouard Bourdelle}

\section{(2) OpenEdition \\ Journals}

Édition électronique

URL : https://journals.openedition.org/aes/4122

DOI : $10.4000 /$ aes. 4122

ISSN : 2258-093X

Éditeur

Laboratoire LISAA

Référence électronique

Édouard Bourdelle, "Usages de la ville, usages du corps : Les Promenades de Paris d'Adolphe Alphand », Arts et Savoirs [En ligne], 16 | 2021, mis en ligne le 17 décembre 2021, consulté le 19 décembre 2021. URL : http://journals.openedition.org/aes/4122 ; DOI : https://doi.org/10.4000/aes 4122

Ce document a été généré automatiquement le 19 décembre 2021.

Centre de recherche LISAA (Littératures SAvoirs et Arts) 


\title{
Usages de la ville, usages du corps : Les Promenades de Paris d'Adolphe Alphand
}

Thinking a new town for a new body: Alphand's Promenades de Paris.

\author{
Édouard Bourdelle
}

1 Les grands travaux haussmanniens, en modifiant la ville, changent les modes de vie des individus. Déplacement des populations les plus pauvres, assainissement hygiéniste de la ville, changement du parc immobilier, avènement de la bourgeoisie, renouveau des pratiques de loisir: tous ces éléments, étudiés par les historiens, montrent que les corps dans la ville subissent des changements profonds. On peut affirmer que le Second Empire consacre un nouveau régime d'usage et de visibilité du corps, dont un des moments privilégiés d'exposition est le temps de la promenade.

2 Cet agencement nouveau n'est pas spécifique à la France. Alain Corbin a très bien montré l'importance du modèle anglais dans le renouvellement des pratiques'. Napoléon III, anglophile, très fortement impressionné par Hyde Park, souhaitait que Paris dispose d'espaces verts qui permettraient à la ville et à ses habitants de respirer : ce vœu a dirigé la création du bois de Boulogne ainsi que celle du bois de Vincennes. Ces espaces ont été entièrement pensés et aménagés par le service des Promenades et Plantations, créé à cette occasion, et confié à Jean-Charles Adolphe Alphand (1817-1891) et à son équipe ${ }^{2}$.

3 Grâce à ces « espaces verdoyants ", selon l'expression de l'époque, l'Empereur imaginait la possibilité d'une mixité sociale, permettant l'harmonie entre les différentes populations de la ville. L'espace vert devait réconcilier les «classes laborieuses $»^{3}$ et la "classe de loisir $»^{4}$ : par son caractère extra-citadin, il devait permettre la jonction entre les deux ceintures de la ville nouvelle. Cette union des corps n'était qu'un vœu pieux, et très vite les deux Bois ont été réservés à une classe particulière de la population: le Bois de Boulogne, à l'aristocratie et la haute bourgeoisie, ainsi qu'au 
passage de l'Empereur ; le Bois de Vincennes, à une certaine bourgeoisie et aux classes populaires. Les deux Bois actent la séparation sociale de Paris entre l'Est et l'Ouest.

Cette séparation est due aux usages propres des populations, comme à la répartition spatiale de la ville, à l'évidence. Cependant, il est aussi probable que leur aménagement singulier ait contribué à ces usages. Par exemple, le travail de Barillet-Deschamps et la création du fleuriste municipal d'Auteuil rendent compte d'une mise en spectacle de la nature, que les promeneurs peuvent contempler et apprécier non seulement pour sa beauté intrinsèque, mais aussi pour sa valeur: les plantes "exotiques", étant rares, coûtent cher. Ce lien entre la valeur de l'objet et le plaisir pris à la contemplation détermine un nouveau rapport, économique, à la promenade, caractéristique d'une haute-bourgeoisie en pleine ascension, et qui vit dans l'Ouest parisien ${ }^{5}$ : celle-ci cherche, même dans l'horticulture, une nature spectaculaire dans un cadre artificiel d'où le développement des serres, dont Zola fera le lieu symbolique de la perversion dans La Curée.

5 Nous trouvons une autre preuve de ce rapport entre aménagement urbain et pratique sociale dans l'ouvrage d'Alphand, Les Promenades de Paris, vaste présentation des quinze années d'aménagement du territoire. Nous proposons de voir comment ce livre, loin de vanter l'utopie sociale de l'Empereur, décrit au contraire une autre répartition de l'espace, beaucoup plus conservatrice. Celle-ci a un caractère prescriptif, qu'il nous faut étudier : elle semble appliquer une nouvelle norme des usages du corps, spécifiques à la ville nouvelle. Nous analyserons d'abord la dimension économique de l'ouvrage, véritable plaidoyer aux aménagements urbains du Second Empire. Ensuite, nous verrons quels usages de la ville le livre d'Alphand intime de suivre, pour enfin rendre compte de la vitrine du mode de vie bourgeois que constitue Les Promenades de Paris.

\section{Les Promenades de Paris, espace publicitaire du Paris du Second Empire}

6 Les Promenades de Paris marquent la fin de la construction de l'ensemble des parcs et jardins de la capitale. Publié entre 1867 et $1873^{6}$, l'ouvrage clôt la vaste entreprise du renouveau de Paris : l'enjeu de l'ouvrage est de placer l'horticulture impériale dans l'Histoire, et de lui donner une place à part, la représentant comme l'aboutissement de toutes les techniques horticoles, au service du progrès et du confort des citadins. Cette dimension téléologique de l'aménagement du territoire est à prendre en considération, d'abord parce qu'elle est voulue par Alphand dans l'édition finale des Promenades de Paris par l'intermédiaire d'une introduction générale qui propose une " histoire des jardins». Alphand se place dans le mouvement historique, et dans une logique positiviste. Il consacre son modèle de jardin comme la synthèse entre l'art et la technique, entre le progrès scientifique et l'expérience artistique, entre le modèle de la nature et le triomphe de l'ingénierie : «Quand nous disons qu'un jardin doit conserver l'aspect de la nature, il ne faut pas croire qu'il s'agit d'une copie exacte des choses qui nous entourent. Un jardin est une œuvre d'art. » ${ }^{7}$

7 Le livre recouvre donc un caractère apologétique. Alphand - ou plutôt ses rédacteurs ${ }^{8}$ doit défendre un projet urbain qui a été extrêmement coûteux, en temps et en argent, en même temps qu'il propose une vitrine de la gloire nouvelle de Paris, par l'intermédiaire de ses jardins. Le succès des Promenades de Paris est surtout 
international: il est un objet publicitaire pour la ville, à destination des touristes comme des habitants. Comme l'écrit Chiara Santini :

La construction des nouveaux jardins donne lieu à une véritable opération de promotion publicitaire et contribue à changer le rapport entre la ville et la proche campagne, ainsi que l'utilisation et la représentation des espaces publics par les habitants. ${ }^{9}$

8 L'objet de cette publication est donc de défendre le projet réalisé selon une triple perspective : technique, esthétique, et sociale.

9 Pour cela, des moyens importants sont proposés par les éditions Rothschild. Le livre paraît en différentes livraisons, avant d'être rassemblé en deux beaux-livres volumineux, avec une illustration très variée. C'est donc un objet d'art, à l'image des jardins qui ont été aménagés. Cela a de quoi étonner: il ne s'agit que d'un livre "technique ", qui devient un livre d'art, achevant l'accès de l'art des jardins à la catégorie des Beaux-Arts ${ }^{10}$. Son enjeu est d'assurer l'hégémonie du génie parisien sur l'art des jardins.

10 Il ne s'agit pas d'une impression issue du pouvoir politique - impérial ou républicain - : c'est même son caractère privé qui a permis à l'œuvre d'être achevée malgré la chute de l'Empire. L'entreprise éditoriale n'obéirait donc pas seulement à l'encomiastique impériale, mais plutôt à une stratégie commerciale: les Promenades de Paris sont publiées pour donner des gages, aux souscripteurs divers, du renouvellement urbain de Paris. Alphand participe à une entreprise de légitimation d'un projet urbain, en même temps qu'il conçoit une réclame de luxe.

11 Le succès du livre est la preuve de l'influence du modèle parisien sur l'art des jardins. Publié à l'occasion de l'Exposition universelle de 1867, Les Promenades de Paris est un succès sans précédent pour un ouvrage "paysagiste", affirmant la puissance symbolique de Paris, capable de concurrencer les grandes villes. Dans toutes les grandes cours d'Europe, comme en province, on trouve des exemplaires des Promenades de Paris : le modèle haussmannien n'est pas qu'urbain, il est aussi paysagiste ${ }^{11}$. Paris devient une référence en matière d'horticulture: à tel point que les ingénieurs d'Alphand conseilleront les ingénieurs responsables de l'aménagement de Central Park, à New-York ${ }^{12}$.

12 Une preuve de cette importance de l'action d'Alphand sur la réputation internationale de Paris se trouve dans l'éloge funèbre que lui consacre la Société des anciens élèves de Polytechnique :

Je connais aussi beaucoup d'autres polytechniciens qui ont été de grands artistes. Je n'en citerai qu'un seul, c'est le plus grand de tous à mon avis.

Je veux parler d'Alphand, Alphand qui a fait le bois de Boulogne avec tous ces paysages délicieux, vrais chefs-d'œuvre que ne désavouerait certainement pas Claude Lorrain; - qui a aménagé artistement et si pratiquement le bois de Vincennes; - qui a fait cet admirable et grandiose décor des Buttes-Chaumont ; qui, en un mot, a tant aimé Paris, et a su en faire la ville superbe et inimitable que vous savez. ${ }^{13}$

13 L'emploi du déictique final est intéressant, puisqu'il atteste comme une évidence l'importance du travail d'Alphand pour la capitale. Cependant, dans cette citation, deux éléments suscitent l'attention: d'abord, la mention d'un espace vert comme "décor " pour le Parisien, qui suppose de la part des contemporains un certain rapport à la nature, un rapport utilitaire aux promenades de Paris qui mettent en avant la magnificence du cadre. L'autre élément est la comparaison qu'opère l'orateur avec la 
peinture du Lorrain. Cette comparaison élogieuse recouvre tout un champ de possibilités, de l'ordre de l'imaginaire, significatif pour comprendre l'usage des promenades par Alphand.

Claude le Lorrain est un peintre du XvII ${ }^{e}$ siècle, reconnu comme un des plus grands paysagistes de l'École Française, avec Poussin. Il représente un sommet de l'art classique pour les hommes et les femmes du XIX siècle, en France et en Angleterre: Ruskin, Turner, Les Goncourt le considèrent comme le plus grand peintre français («Lorrain a tiré un feu d'artifice dans les décors de Poussin $»^{14}$ ). Jacques Rancière montre même comment, au XIXe siècle, les théoriciens du jardin ont trouvé leur source d'inspiration chez ce peintre, proposant « une réforme du regard et non une méthode effective pour aménager les parcs $\aleph^{15}$. Sa peinture, mêlant ruines et paysages, a trouvé un écho particulier chez les romantiques, en même temps que son travail de la lumière a été admiré par une sensibilité « moderne » du milieu du XIX ${ }^{\mathrm{e}}$ siècle : Millet, Van Gogh, Rousseau, sont des admirateurs de la peinture du Lorrain. Comparer Alphand à un peintre classique de cette envergure, c'est donc donner au jardin la place d'un art à part entière.

15 En même temps, la peinture du Lorrain dévoile un imaginaire particulier : la petitesse des hommes face au spectacle de la nature, dans ses peintures, fait des individus des spectateurs d'une nature sublime, offrant une méditation sur l'action des hommes. Cette peinture offre des espaces de tranquillité au spectateur. La nature y devient un spectacle : or Alphand pense l'organisation de son paysage selon un même besoin d'une nature spectaculaire, dans un usage proche de celui du Lorrain. En effet, la promenade, dans son ouvrage, est un espace de méditation et de repos, un espace de loisir sain à l'abri de l'activité industrielle : il présente souvent l'espace verdoyant comme un locus amoenus. Et son éloge du jardin d'Ermenonville ${ }^{16}$, dans son introduction, indique sa vision de son art : la végétation donne plusieurs occasions à des points de vue notables, et Alphand décrit de façon très précise les effets de lumière et de peinture que la sensation du végétal doit créer sur l'œil humain. Le parc d'Ermenonville crée la désorganisation, qui permet au promeneur de se perdre dans l'espace, sans pour autant engendrer la confusion, qui nuit à sa tranquillité.

Ce risque de confusion explique sa critique du jardin « romantique » qui, en multipliant les outils de décoration jusqu'à la saturation, et en délaissant la surprise de la nature, pervertit le projet paysagiste. Sa critique de la monumentalité va dans le même sens, car celle-ci ne permet pas une véritable station de promenade: on ne sait plus où s'arrêter avec la multiplication des bâtiments artificiels et des fausses ruines ${ }^{17}$. De fait, le jardin romantique fatigue, alors que le jardin d'Alphand est avant tout un espace de délassement. Dire de l'œuvre d'Alphand qu'elle se rapproche de celle du Lorrain, c'est mettre en avant un gigantisme de la nature, qui impose le silence et l'admiration à celui qui la regarde : c'est une certaine idée de la promenade, et de l'espace vert, qui se dégage, où le corps du promeneur est un corps en repos, adonné au loisir d'un spectacle qui le fait sortir de lui-même.

En effet, la peinture du Lorrain se caractérise par trois aspects: une perspective rigoureuse, une nature monumentale prenant le pas sur la ville, des individus toujours minuscules dans cette coexistence de la nature et de la ville, spectateurs du monde qui les entoure. Exactement l'image de la nature qu'Alphand illustre dans Les Promenades de Paris. Nous en voulons pour exemple la dernière illustration de la section des «Promenades intérieures ${ }^{18}$ qui reprend ces trois caractéristiques distinguées chez 
Lorrain. Le corps du promeneur, on le voit, y est entièrement adonné à la contemplation : au centre du premier plan, les deux personnages sont pris dans un paysage luxuriant, qui a pour fonction de les impressionner et de les dépayser. Le corps «dans la ville» semble paradoxalement «sorti » de celle-ci: le square a pour effet d'extraire le promeneur de son paysage habituel.

Fig. 1

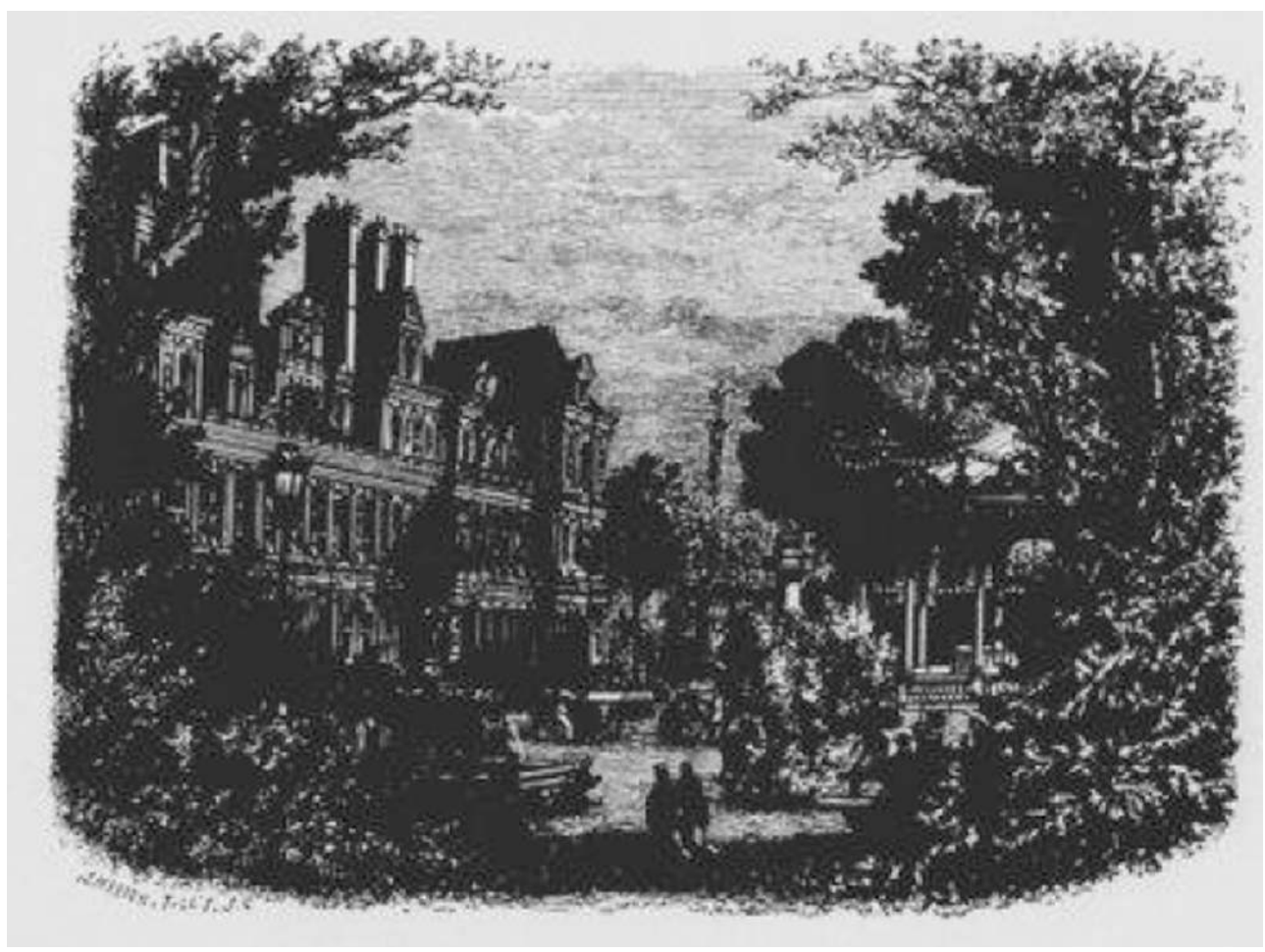

Square des Batignolles, détails. Source : Jean-Charles Adolphe Alphand, Les Promenades de Paris, «les Promenades intérieures de Paris, op. cit. p. 246.

Ici, on se trouve comme à l'orée d'un bois, endroit privilégié de l'observateur. Le cadre végétal est intéressant, car il impose une concentration du regard : l'illustration nous permet de contempler la coexistence de la ville et de l'espace vert, en même temps qu'elle montre une vision complètement chimérique de la ville.

19 L'image proposée est imaginaire. Il s'agit du Square des Batignolles, où un bâtiment imposant est encerclé de verdure. Le décor au premier plan semble nous isoler du monde: il crée un espace vert autarcique pour ces deux promeneurs, dont nous épousons le point de vue. Entourés par une nature foisonnante, qui envahit littéralement l'espace visuel, une perspective étrange est construite : on semble sortir d'un bois en arrivant dans la ville, comme le montrent les arbres très hauts qui se développent de part et d'autre des promeneurs. Or, ces arbres n'existent pas, en réalité : le square est bien entouré d'arbres, mais ceux-ci n'ont pas ce foisonnement enveloppant. On crée, dans la fiction, un espace à la fois d'isolement (le premier plan) et de communication avec la ville (l'arrière-plan). Le chapitre se clôt par l'image fantasmée d'un Paris qui serait devenu entièrement promenade, d'une ville faite de réseaux, tout entière faite pour être parcourue : un mode de vie, celui du promeneur dans sa ville, semble ainsi être affirmé dans le livre. 
20 Ce type d'illustration n'est pas isolé dans l'ouvrage. À plusieurs reprises, celles-ci montrent un Paris qui est celui imaginé par Alphand, mais guère celui véritable. Elles expliquent le but du livre, qui est de "donner à rêver" Paris. On le voit dans les chapitres consacrés au Parc Monceau et aux deux Bois en particulier, où tous les outils sont utiles pour souligner le caractère extraordinaire du lieu: Alphand doit frapper l'imaginaire du lecteur et du spectateur, afin d'avoir son accord tacite par l'étonnement. Il s'agit d'une stratégie de persuasion, qui cherche à travailler avec l'imagination : le lecteur doit savoir s'imaginer dans les espaces qui lui sont exposés. On lui présente donc des individus qui lui ressemblent, dans des promenades, ou des points de vue qu'il pourra voir : implicitement, donc, des corps normés apparaissent, avec des points de vue et des regards spécifiques, qui ont pour effet d'uniformiser l'expérience de la promenade dans Paris selon un type d'utilisateur. Il est significatif, en effet, que toutes les illustrations reprennent une forme particulière de promenade: Alphand propose donc un mode de vie, sous couvert de défendre une œuvre urbaniste. Tout en donnant à voir un Paris pour les touristes, c'est bien l'imposition d'un nouvel usage de la ville pour ses habitants qui est illustré.

\section{Usages de l'espace : Les Promenades de Paris et les nouveaux usages de la ville}

21 " Policée, cette architecture verte, qui ne relève pas tout à fait de la ville construite ni de la nature libérée, vise avant tout le plaisir de l'œil. ${ }^{19}$ On a beaucoup insisté sur la valeur décorative de l'espace vert. Cependant, le Baron Ernouf, héritier d'Alphand, explique dans son Art des Jardins que cet espace n'obéit pas qu'à un usage esthétique. Ces considérations artistiques ont un but social, où l'hygiénisme revêt un caractère normatif :

Les squares et les autres jardins de Paris n'ont pas été faits seulement pour le plaisir des yeux. Sans doute la question d'art eût suffi à justifier ces créations, mais les jardins publics dans les villes sont des œuvres non moins utiles qu'agréables. Quand on pense à cette énorme agglomération parisienne, [...] on doit considérer comme une nécessité la formation de larges voies et de surfaces plantées, assez spacieuses, assez rapprochées pour ventiler ces masses de pierres qui semblent percées de couloirs étroits, la largeur des rues étant moindre que la hauteur des maisons.

Autrefois, pour respirer un peu d'air pur et jouir du soleil, il fallait, ou gagner des promenades éloignées, ou même sortir de Paris; aujourd'hui, les Parisiens ont à leur disposition des jardins répartis à peu près également dans tous les quartiers de la Ville. ${ }^{20}$

Les transformations de Paris ont obéi à une nécessité, en même temps qu'elles ont cherché à améliorer la vie de ses habitants. Le Beau n'y est nullement accessoire : il obéit à une fonction morale. Alphand, à la fin de son introduction des Promenades de Paris, ne dit pas autre chose :

Mais depuis longtemps, même dans les localités où les populations sont le moins adonnées au travail industriel, les rues et les ruelles sont devenues insuffisantes, et les larges voies ont été imposées par le développement énorme de la circulation moderne. Elles ont été plantées, autant pour donner de l'agrément que pour introduire dans les villes un élément de salubrité. Ces avenues, où sont disposés des bancs invitant au repos, où le passant trouve de l'ombre pendant l'été, sont encore trop peu nombreuses, même à Paris, qui a pris l'initiative de ces améliorations et donné l'exemple de la transformation des vieilles cités, non en réalisant des cuvres de fantaisie et de vain faste 
comme dans l'antiquité, mais en appliquant les conquêtes de la science et de l'art à

la viabilité et à la salubrité de la grande cité. ${ }^{21}$

"Salubrité », « repos », " ombre », sans «fantaisie » ni «faste » : la dimension sociale des jardins publics n'est pas à minimiser. «Le réseau des « espaces verdoyants » répond aux soucis d'ordre social de Napoléon III, mais aussi à des préoccupations sanitaires : offrir des espaces de détente et de loisir aux Parisiens ${ }^{22}$. On y vante un loisir socialement utile. Nombreux sont les historiens à rappeler que Napoléon III, quand il initie les grands travaux, croit en l'utopie sociale du rassemblement des populations. Alphand reprend cet argumentaire, quand il explique l'utilité du Parc Montsouris :

Établi sur un plateau légèrement ondulé, qui domine la vallée de la Bièvre, et d'où l'œil embrasse une vue de Paris fort étendue, le parc de Montsouris est destiné à appeler sur la rive gauche de la Seine, comme les Buttes Chaumont sur la rive droite, la vie et le mouvement au milieu de quartiers précédemment voués à l'isolement et à l'abandon. ${ }^{23}$

Et la pensée sociale des ingénieurs des Ponts et Chaussées - où Alphand a suivi sa formation - est du même ordre. Comme le dit Antoine Picon :

Sous le Second Empire, cette orientation politique inspirait des entreprises comme les plantations de Paris [...]. Implanté au cœur d'une des zones les plus défavorisées de la capitale, le parc des Buttes-Chaumont constitue l'une des expressions les plus frappantes de ce projet. S'il n'était pas question d'enrichir les pauvres ou d'appauvrir les riches, les uns et les autres devaient pouvoir accéder à une nature disciplinée par les jardiniers et les ingénieurs des Ponts et Chaussées. ${ }^{24}$

L'agrandissement de Paris suppose de travailler sa cohésion : il faut unifier la capitale. Chiara Santini explique que toute l'entreprise haussmannienne repose sur la création de réseaux pour relier les différentes parties de la ville entre elles ${ }^{25}$. Cette image du réseau est aussi à comprendre dans son sens social : le jardin est pensé comme un espace de mixité sociale, et doit empêcher l'isolement des populations laborieuses. Tout "vide» dans les grands travaux est remplacé par un parc, un square, etc. Nous assistons à l'usage d'une "normalisation " de la verdure où on comble d'arbres tout espace vide. La lecture du plan de Paris sous Alphand et Haussmann montre bien que la nature haussmannienne a horreur du vide, et il faut dès lors absolument investir les espaces, selon une fonction sociale d'hygiénisme et de purification par le loisir : acheter une " paix sociale" par l'espace vert. Toujours à la fin de son introduction, Alphand rallie cette dimension hygiéniste héritée des saint-simoniens, à une téléologie du progrès scientifique. Au premier abord, les travaux parisiens n'ont d'autre but que de prévenir la maladie. L'hygiénisme est biologique :

Plus la science progresse, plus les hommes sont attentifs à réaliser les meilleures conditions hygiéniques, au dedans et aux abords des habitations. ${ }^{26}$

Ces propos ne sont pas sans rappeler les projets philanthropiques de Godin (et son Familistère), pour qui les meilleures conditions hygiéniques étaient garantes de l'amélioration morale des ouvriers. Godin, dans ses Solutions Sociales ${ }^{27}$ théorise même les « équivalents de la richesse », à savoir l'air pur, l'espace libre, la lumière, la salubrité et l'hygiène; richesses qui auront une conséquence sociale. Comme l'écrit Jessica Dos Santos à propos des projets le précédant, et qu'il critique :

Il [Godin] estime que ceux-ci [les projets de corons dans le Nord] n'ont en réalité eu pour but que de déplacer les ouvriers de leur village vers la proximité des mines, sans rien changer à leur mode de vie, tandis que la cité de Mulhouse, par ses appartements et ses jardins trop petits, a réussi à cumuler les défauts de la maison isolée et de la promiscuité. Ces deux expériences seraient donc, aux yeux de Godin, 
sans aucun intérêt si elles n'avaient été accompagnées par la création d'institutions d'utilité commune, assurant hygiène et salubrité, ainsi que la possibilité d'une scolarisation pour les enfants. ${ }^{28}$ d'abord biologique. Cependant, il est évident que du point de vue du grand projet haussmannien, cet hygiénisme a des conséquences psychologiques et morales. Géraldine Texier-Rideau va même plus loin, insistant sur la norme d'usage qu'imposent les pouvoirs publics, quand ils refusent de faire des espaces verts des espaces de traversée :

Cette dernière requête, toujours refusée par le service des Promenades - qui considère que le square n'est pas un espace de traversée mais d'arrêt -, révèle une mutation plus profonde qui dépasse largement le cadre d'un aménagement: il touche aussi la pratique même de l'espace public. Investie en son centre, fermée, policée, la place des anciens villages ne joue plus le même rôle. Elle n'articule plus l'espace public, se concentre sur l'espace vert qui, superposé, lui fait perdre jusqu'à son identité. [...] Le square réinventé est devenu une « anti-place de circulation $» .^{29}$

voit ici la double articulation de la construction des espaces verts parisiens: il s'agissait d'augmenter les possibilités pour les habitants de se reposer à l'intérieur de la ville, en transformant radicalement leurs usages de celle-ci. C'est-à-dire qu'en transformant la capitale, en l'élargissant, les espaces verts augmentent aussi le champ de contrôle qu'elle représente : car ces jardins publics sont autant d'espaces surveillés par la ville de Paris. Ne pas sortir de Paris pour « respirer » revient à contrôler le loisir des Parisiens. Emmanuel Pernoud rappelle que Vallès critiquait ces espaces verts pour cette raison précise que la " paix sociale " y était une paix « imposée », et nullement celle d'un loisir « gratuit» :

Pour Jules Vallès, le jardin public n'est pas une clairière, un havre de paix ; c'est une soupape ou une tombe, cela sert à maintenir l'ordre social ou à enterrer les crimes. Le silence et le vide ne sont pas le résultat d'un allègement, d'un oubli des affaires du monde, mais d'une oppression parmi d'autres, d'un écrasement artificiellement remporté, contre la conscience d'être écrasé. ${ }^{30}$

Il faut donc voir en quoi Les Promenades de Paris nous donne justement des espaces de contrôle des corps, ou laissent envisager de la part d'Alphand la livraison de l'image d'un certain Paris, pensé selon un loisir bourgeois. Il s'agit d'un Paris en sécurité, qui ici n'a plus pour seule fonction de prévenir la maladie, mais bien d'assurer le calme de la cité. Une nature urbaine d'un nouveau genre y est défendue, qui s'éloigne des loisirs de la campagne :

La nature qui les décore n'est pas une nature productive - synthèse ou maquette de la campagne environnante - mais une nature complètement artificielle, une mise en scène raffinée et hautement technique de paysages lointains. Cet aspect du projet haussmannien lance une redéfinition importante du rapport ville campagne. Il coupe définitivement les liens historiques entre sites de production et lieux de consommation des produits agricoles. Il efface les trames vertes, discontinues et étriquées au travers desquelles, à l'époque moderne, la campagne s'insérait dans le tissu urbain et par lesquelles la ville se reliait à la campagne. Désormais, deux natures et deux mondes s'opposent. D'un côté, il y a la ville, avec ses parcs pittoresques ornés d'essences rares et ses longues promenades où les arbres semblent pousser du ciment, droits et bien alignés. De l'autre côté, il y a la campagne : une campagne à la fois très proche et lointaine. Elle se caractérise par une autre nature : celle productive des champs et des grandes exploitations et celle, investie par la rêverie citadine de l'époque industrielle, des bois silencieux et des eaux ruisselantes où, le dimanche, s'animent guinguettes et cercles des canotiers. ${ }^{31}$ 

sur l'analyse du bois de Vincennes. Celui-ci nous intéresse davantage que le bois de Boulogne, puisque ses usagers, à l'époque d'Alphand, sont d'origine plus modeste et populaire que ceux de l'Ouest parisien. Les îles du bois de Vincennes offrent des plaisirs populaires, mais Alphand les mentionne de façon allusive : les illustrations consacrées à Vincennes mettent en avant les promenades amoureuses en barque, sur le lac. Il est intéressant de voir comment le texte, s'il mentionne les fêtes populaires, tend à les effacer, pour vanter les mérites du Bois de Vincennes comme un bois pour « promeneur solitaire » ou « romantique ».

31 Le bois de Vincennes, en effet, est boudé par l'aristocratie parisienne, qui préfère le bois de Boulogne et les rites qui lui sont associés. Tout un cérémonial se déploie dans la pratique de la promenade au Bois ${ }^{32}$, où une sociabilité festive de la distinction s'impose : la distance est le propre de la sociabilité impériale, où l'on évite tout contact. À l'inverse, le bois de Vincennes, avec ses îles, est propice aux loisirs plus populaires :

La sociabilité qui s'y déploie n'a pourtant que peu à voir avec le bois de Boulogne : l'un est animé, bruyant, étincelant tous les jours de la semaine, l'autre aménagé dans la continuité du laborieux boulevard de la Bastille, à proximité d'une ville de Vincennes réputée pour sa laideur et sa malpropreté, affiche le paradoxe d'une gaieté terne et triste parce que populaire. Peu de ces commerces du divertissement qui font l'animation du Bois de Boulogne s'y installent. ${ }^{33}$

Cette désaffection s'explique aussi par l'intervention d'Alphand, qui établit un règlement très strict, en particulier sur tout ce qui pourrait dénaturer le plaisir de la promenade. Ainsi est-on sensible, chez Alphand, à cette volonté d'empêcher la construction de lieux de réunions publiques, de restaurants ou commerces, de lieux exhalant des odeurs désagréables, d'affiches et de publicités, bref : de tout ce qui pourrait empêcher la promenade d'avoir lieu ${ }^{34}$.

L'argumentaire d'Alphand est toujours lié à une idée préétablie de la promenade : un «plaisir » rousseauiste, qui permet d'oublier le quotidien. En un sens, la promenade rejoint la notion d'otium antique (temps libre de l'inaction), opposée au negotium (temps de l'activité industrieuse). On comprend son éloge du jardin d'Ermenonville, précédemment mentionné : la technique du paysagiste est au service de l'aménagement d'un espace de repos. La promenade offre l'accès à un moment d'oubli, presque à un «temps mort».

On comprend qu'Alphand parvient toujours à donner une raison pratique à un aménagement technique. Il y a vraiment un lien net entre la technique du jardin et la technique du corps qui s'ensuit. Par exemple, il explique comment amener un promeneur à marcher «naturellement » dans le jardin : il refuse la ligne droite pour une allée.

II faut que le paysage change d'aspect à mesure que l'on se déplace; c'est encore une raison qui doit faire proscrire la ligne droite dans les jardins pittoresques, indépendamment de tous les autres motifs qui imposent son abandon. La ligne courbe force le promeneur à se déplacer latéralement, et la ligne de vue est toujours tangentielle à la courbe de l'allée; par conséquent, le tableau change constamment d'aspect durant la promenade. Le tracé d'une allée ne doit donc jamais être parallèle à l'axe d'une ligne perspective, à moins qu'on ne veuille prolonger la durée de la vision dans une même direction. ${ }^{35}$

De la même manière, on peut être sensible à sa description du percement du lac de Saint-Mandé, pour le Bois de Vincennes : 
Un second lac fut creusé près de Saint-Mandé, sur l'emplacement d'une ancienne retenue de moulin, qu'entretenait le ru de Montreuil. Ce lac a une superficie d'un hectare et demi environ, non compris la surface de l'ile conservée au centre. Placé au fond d'une vallée verdoyante, entouré de routes sinueuses, encadré de plantations magnifiques, alimenté par des ruisseaux aux capricieux méandres, retombant çà et là en chutes sonores, le lac de Saint-Mandé est une des parties les plus pittoresques de la nouvelle promenade. ${ }^{36}$

Ce passage est intéressant, car il tente de rendre compte littérairement du caractère pittoresque de cette promenade. Les adjectifs employés ici ne sont nullement neutres; soit ils relèvent de l'hyperbole, soit ils révèlent un art de la correspondance, voire de la métaphore (les capricieux méandres, retombant çà et là en chutes sonores, rappellent la théorie de Baudelaire). L'intérêt de cette petite phrase est de se demander quelle valeur elle a: outre la question de la légitimation de la promenade par les sens et l'imagination, et de la construction culturelle d'une émotion esthétique, dans ce passage l'architecte se laisse emporter par la beauté de sa création, allant à la création d'un tableau à l'intérieur de son texte, afin de montrer, ici, par l'exemple, l'art du paysagiste: la création d'un tableau vivant. Cette description a quasiment la valeur d'une hypotypose, puisque les termes tentent de rendre compte d'un dynamisme du paysage, afin de vanter les mérites du Bois pour un promeneur solitaire.

On peut analyser la page qui suit de la même manière ${ }^{37}$, autant pour son texte que pour ses illustrations : les deux images proposent un point de vue, un paysage à contempler, à même le livre, comme une invitation à la promenade. Ces illustrations sont intrigantes: elles donnent à voir une nature luxuriante, sans présence humaine aucune, comme si le lac de Charenton était un endroit à contempler, avant d'être un lieu de sociabilité. On retrouve ici le luxe, le calme et la volupté vantés par Alphand, qui pense l'espace de la promenade comme un espace de repos et d'évasion. Dans la page, entre deux images, on trouve la mention directe de « touristes » : 
Fig. 2

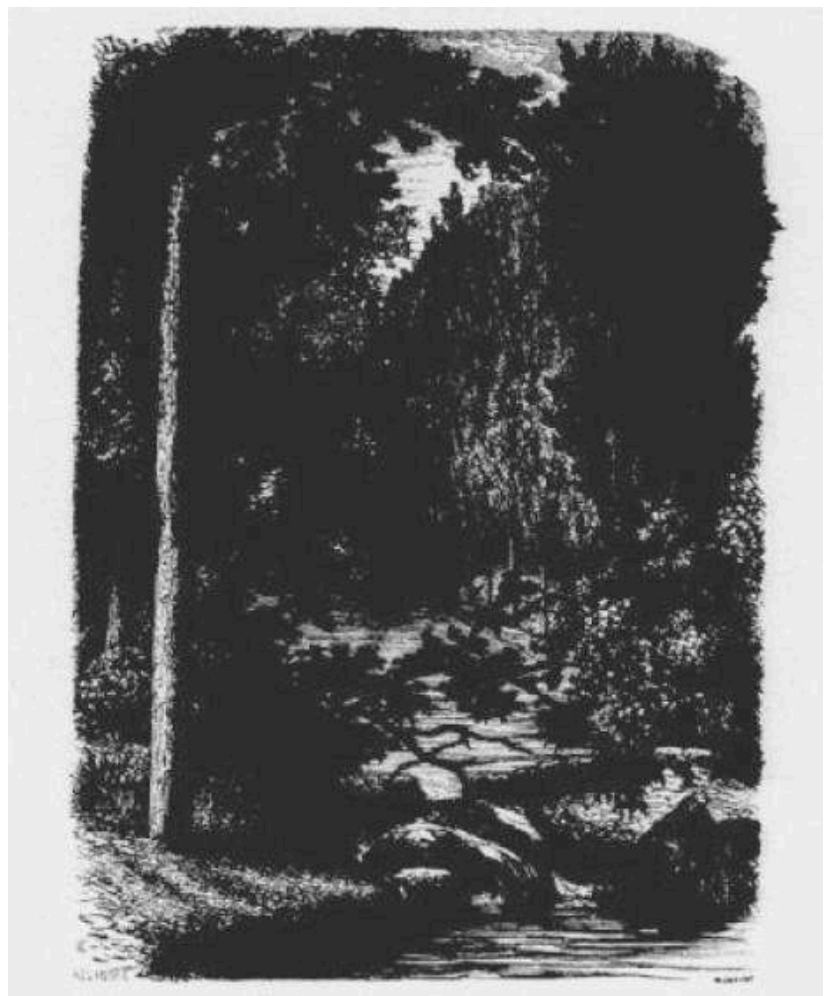

Barrage de la Rivière de Charenton. Source : Jean-Charles Adolphe Alphand, Les Promenades de Paris, «le Bois de Vincennes », op. cit. p. 164

Du haut de cette butte, on jouit d'un admirable panorama. Souvent les touristes vont chercher bien loin des points de vue renommés, qui n'égalent pas celui de Gravelle, dont le tort unique est de se trouver à la porte de Paris, et d'être d'un abord aussi facile que rapide.

Fig. 3

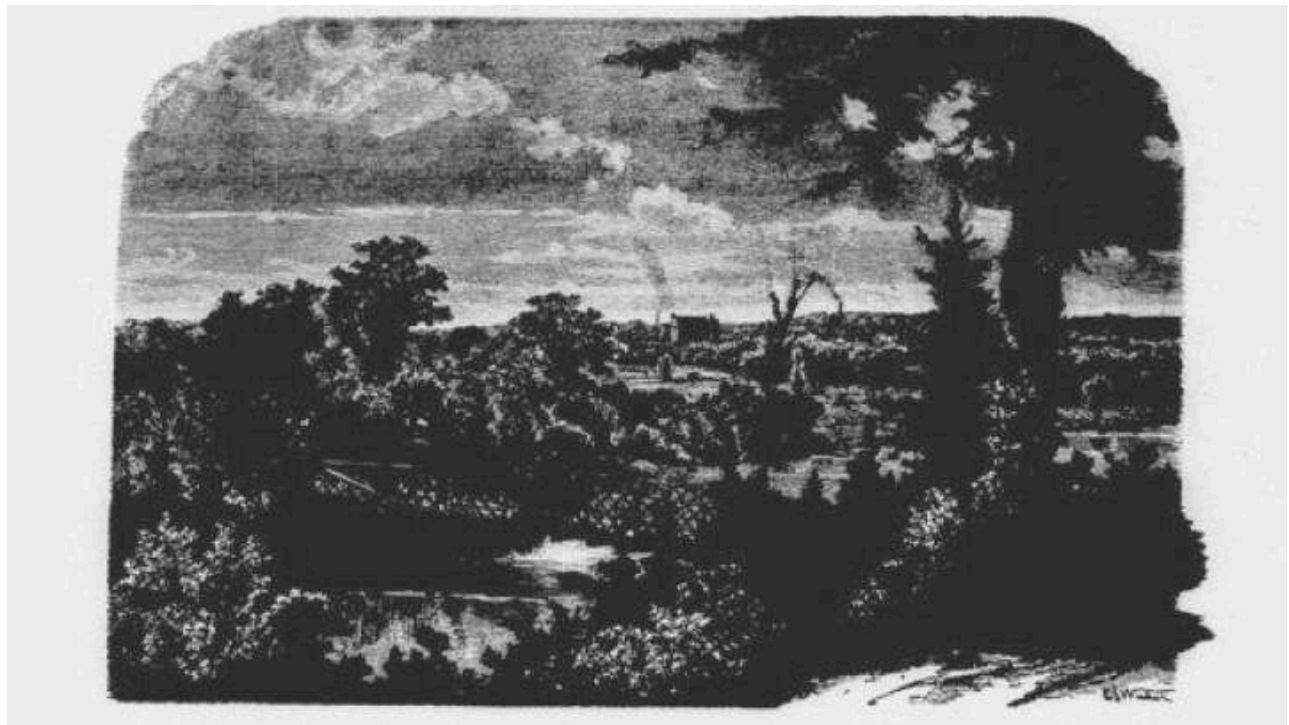

Vue du pont suspendu sur le Lac de Charenton.. Source : Jean-Charles Adolphe Alphand, Les Promenades de Paris, «le Bois de Vincennes», op. cit. p. 164 
Les pages qui ont précédé ont mentionné l'insistance d'Alphand sur le temps du repos,
et sur la création d'espaces de pause. De façon significative, on voit qu'une rupture
s'établit entre le «loisir » et le «plaisir »; le loisir est désormais le temps du repos, du
"temps mort », et non plus celui du plaisir pris, que l'on pourrait qualifier de «temps
de vie ». Ce temps du repos, de l'intime, trouve son illustration dans Les Promenades de
Paris. Par exemple la gravure suivante fait de l'espace public un espace intime, voué à
l'occupation familiale:

Les pages qui ont précédé ont mentionné l'insistance d'Alphand sur le temps du repos,
et sur la création d'espaces de pause. De façon significative, on voit qu'une rupture
s'établit entre le «loisir » et le «plaisir »; le loisir est désormais le temps du repos, du
"temps mort », et non plus celui du plaisir pris, que l'on pourrait qualifier de «temps
de vie ». Ce temps du repos, de l'intime, trouve son illustration dans Les Promenades de
Paris. Par exemple la gravure suivante fait de l'espace public un espace intime, voué à
l'occupation familiale:

Les pages qui ont précédé ont mentionné l'insistance d'Alphand sur le temps du repos,
et sur la création d'espaces de pause. De façon significative, on voit qu'une rupture
s'établit entre le «loisir » et le «plaisir »; le loisir est désormais le temps du repos, du
"temps mort », et non plus celui du plaisir pris, que l'on pourrait qualifier de «temps
de vie ». Ce temps du repos, de l'intime, trouve son illustration dans Les Promenades de
Paris. Par exemple la gravure suivante fait de l'espace public un espace intime, voué à
l'occupation familiale:

Les pages qui ont précédé ont mentionné l'insistance d'Alphand sur le temps du repos,
et sur la création d'espaces de pause. De façon significative, on voit qu'une rupture
s'établit entre le «loisir » et le «plaisir »; le loisir est désormais le temps du repos, du
"temps mort », et non plus celui du plaisir pris, que l'on pourrait qualifier de «temps
de vie ». Ce temps du repos, de l'intime, trouve son illustration dans Les Promenades de
Paris. Par exemple la gravure suivante fait de l'espace public un espace intime, voué à
l'occupation familiale:

Les pages qui ont précédé ont mentionné l'insistance d'Alphand sur le temps du repos,
et sur la création d'espaces de pause. De façon significative, on voit qu'une rupture
s'établit entre le «loisir » et le «plaisir »; le loisir est désormais le temps du repos, du
"temps mort », et non plus celui du plaisir pris, que l'on pourrait qualifier de «temps
de vie ». Ce temps du repos, de l'intime, trouve son illustration dans Les Promenades de
Paris. Par exemple la gravure suivante fait de l'espace public un espace intime, voué à
l'occupation familiale:

Les pages qui ont précédé ont mentionné l'insistance d'Alphand sur le temps du repos,
et sur la création d'espaces de pause. De façon significative, on voit qu'une rupture
s'établit entre le «loisir » et le «plaisir »; le loisir est désormais le temps du repos, du
"temps mort », et non plus celui du plaisir pris, que l'on pourrait qualifier de «temps
de vie ». Ce temps du repos, de l'intime, trouve son illustration dans Les Promenades de
Paris. Par exemple la gravure suivante fait de l'espace public un espace intime, voué à
l'occupation familiale:

Les pages qui ont précédé ont mentionné l'insistance d'Alphand sur le temps du repos,
et sur la création d'espaces de pause. De façon significative, on voit qu'une rupture
s'établit entre le «loisir » et le «plaisir »; le loisir est désormais le temps du repos, du
"temps mort », et non plus celui du plaisir pris, que l'on pourrait qualifier de «temps
de vie ». Ce temps du repos, de l'intime, trouve son illustration dans Les Promenades de
Paris. Par exemple la gravure suivante fait de l'espace public un espace intime, voué à
l'occupation familiale:

Comme les gravures de mode, les illustrations du livre d'Alphand exposent donc un usage modèle des jardins et des squares : il ne s'agit pas de représentations (venant après ce qu'elles présentent), mais de projections, quand bien même les planches seraient-elles réalisées après les lieux qu'elles dépeignent. Alphand propose au lecteur l'image idéale qu'il se fait de ses jardins après leur ouverture, il confie à ces vues un caractère didactique qui en fait plus que des descriptions : illustrations qui nous disent ce que l'on doit faire d'un square, comment s'y tenir, comment y bouger, qui suggèrent aussi le genre de personnes à qui le square est destiné. ${ }^{38}$

\section{Les Promenades de Paris, triomphe de la bourgeoisie?}

Les illustrations que nous avons analysées étaient de l'ordre d'une projection, et renvoyaient à l'usage pensé par Alphand de ses promenades. Cependant, aucun être humain "réaliste» ne s'en dégage. À lire les écrivains de l'époque, la réalité des promenades parisiennes est tout autre, et l'on voit que plusieurs imaginaires sont en concurrence : celui de l'aristocratie impériale, qui fréquente le bois de Boulogne pour parader ; celui de la bohême, qui y tue son ennui ${ }^{39}$. 
Fig. 4

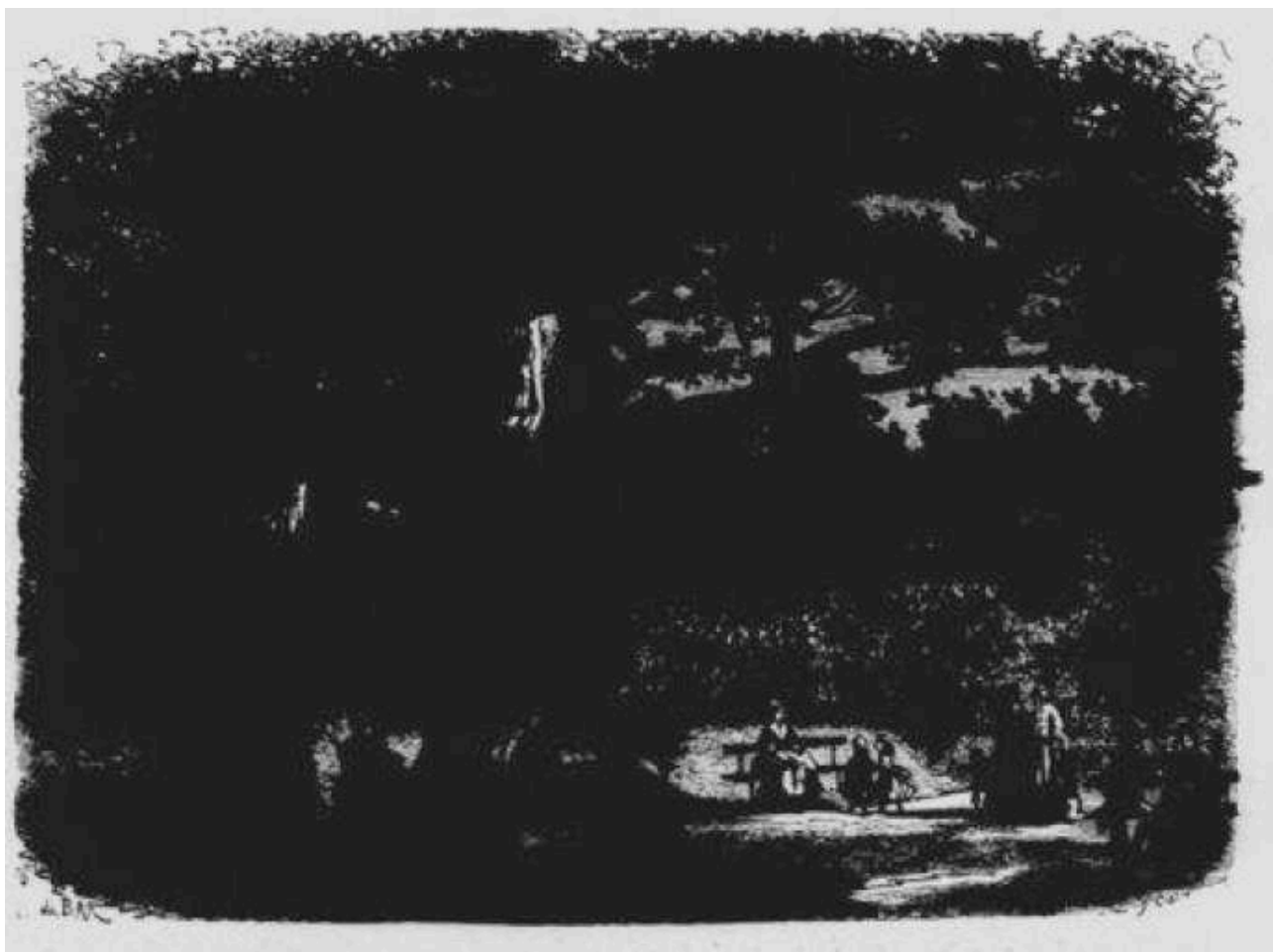

Vieux chênes d'Auteuil. Source : Jean-Charles Adolphe Alphand, Les Promenades de Paris, «le Bois de Boulogne», op. cit. p. 38

43 La gravure suivante fait de l'espace technique (un «barrage ») un espace érotique celui de la première rencontre amoureuse dans un cadre bucolique -, mais d'un amour chaste, loin de l'usage réel du Bois : 
Fig. 5

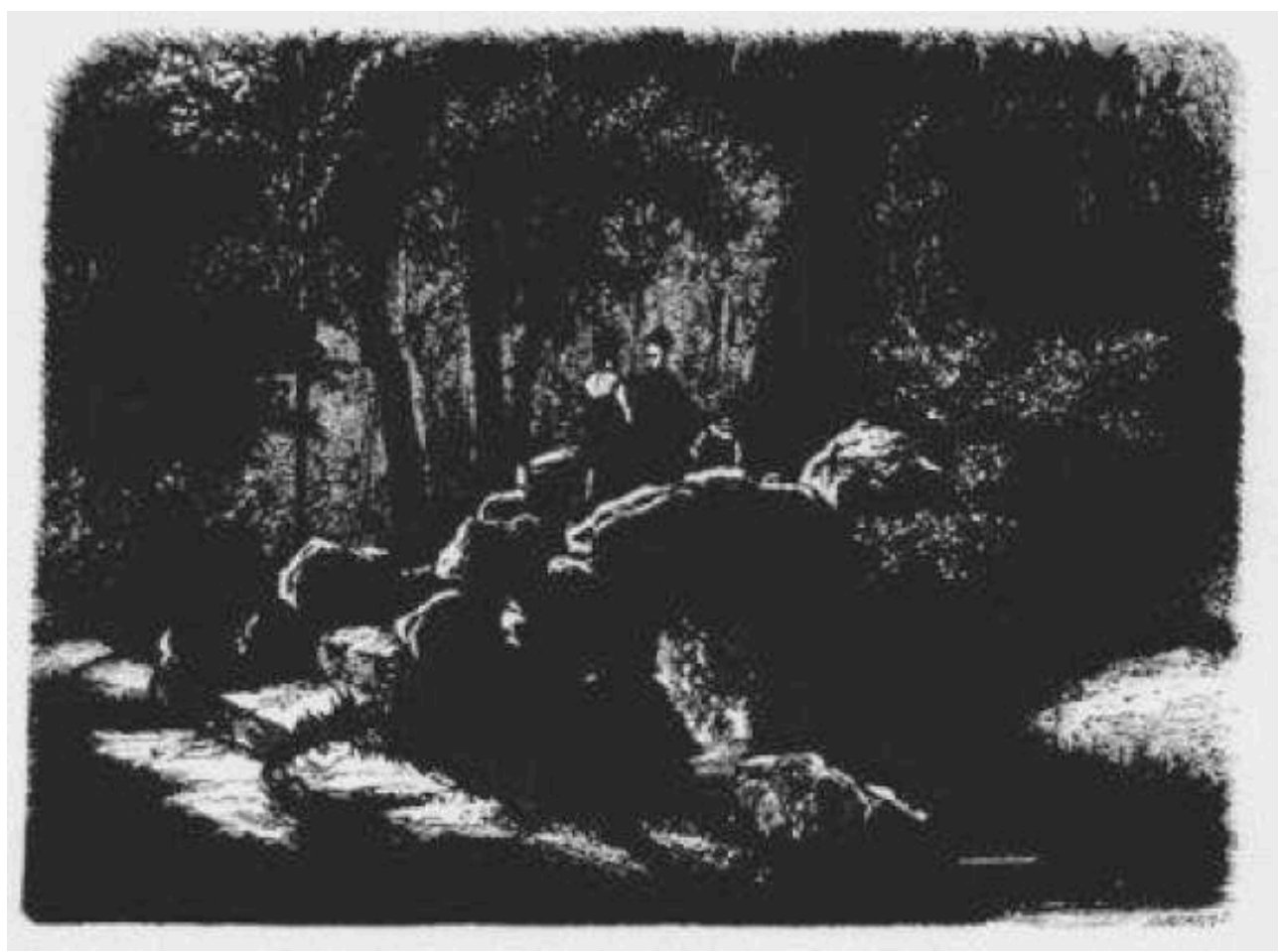

Barrage sur le ruisseau de Longchamps (sic). Source : Jean-Charles Adolphe Alphand, Les Promenades de Paris, «le Bois de Boulogne», op. cit. p. 55 nécessité du repos. Beaucoup de héros sont des "surmenés » ou des "névropathes », qui s'isolent de la grande ville pour se reconstruire. Ainsi Charles Demailly, dans le roman éponyme des Goncourt, doit-il quitter la capitale et se réfugier à Troyes après une crise. Il est significatif les propos qui suivent, la neurasthénie, soient prononcés par un médecin : celui-ci donnerait une autre justification à l'aménagement horticole dans Paris, de contrôler des malades du siècle :

\begin{abstract}
Moi, je la regarde [la neurasthénie], au contraire, comme une maladie organique et propre, au moins par ses caractères de généralité et d'excès, à la race du XIX ${ }^{\mathrm{e}}$ siècle. Je la crois le mal de tous les habitants des capitales, à des degrés morbifiques différents, mais compromettant du plus au moins la santé des générations à naître ; car des forts naissent les forts... Et regardez, tout se précipite à la centralisation, à la formation de grandes et de petites capitales. La vie moderne va du plein air de la vie agricole à la vie concentrée, à la vie assise, à la vie au gaz du charbon de terre, à la vie au gaz des lampes, à la vie nourrie par une alimentation falsifiée, sophistiquée, trompeuse, à tous les renversements des conditions normales de l'être physique [...] Et le temps presse, monsieur, il n'y a pas à s'y tromper. [...] Tous, nous sommes surexcités... et jusqu'à nos enfants dont nous poussons l'esprit qui bégaye comme on pousse une plante en serre chaude. C'est une circulation fiévreuse de la vie, une irritation, presque une crise, de tout ce qui est la partie délicate et comme immatérielle de notre individu... ${ }^{40}$
\end{abstract}

Ce discours nous permettrait de comprendre l'enjeu urbanistique des aménagements parisiens, comme des espaces de repos et d'intimité, des espaces qui font oublier la ville.

Ce qui s'instaure, c'est une idéologie du temps mort, qu'il nous faut désormais étudier. La notion même de «plaisir » est condamnée avec la démocratisation des loisirs. Par 
exemple, dans leur Journal, les frères Goncourt, au début de l'année 1857, se montrent critiques vis-à-vis d'un monde en train de se démocratiser :

18 janvier. - Été hier au bal masqué. Voici une chose grave, plus grave qu'on ne croit: le Plaisir est mort. Ce rendez-vous de l'imprévu, ce coudoiement de rencontres, cette foire de romans d'aventure, ce feu roulant de reparties, ce carnaval de la gaieté et de l'amour, cette folie, cette joie démente d'une jeunesse furieuse, qui sautait douze heures sous l'archet de Musard, la fouettant et la refouettant des fifres et des tonnerres de son orchestre: ce n'est plus tout cela qu'un trottoir. ${ }^{41}$

Quand on regarde l'illustration suivante de la Croix-Catelan, un des endroits les plus enjoués du Bois de Boulogne, où était situé un théâtre en plein air, et où s'organisaient des bals, nous pouvons comprendre le regret des Goncourt. L'espace y est mieux découpé, plus large - il n'y a pas de " coudoiement ", et les costumes des hommes et des femmes, s'ils sont élégants, ne sont nullement ceux d'usage pour une fête :

Fig. 6

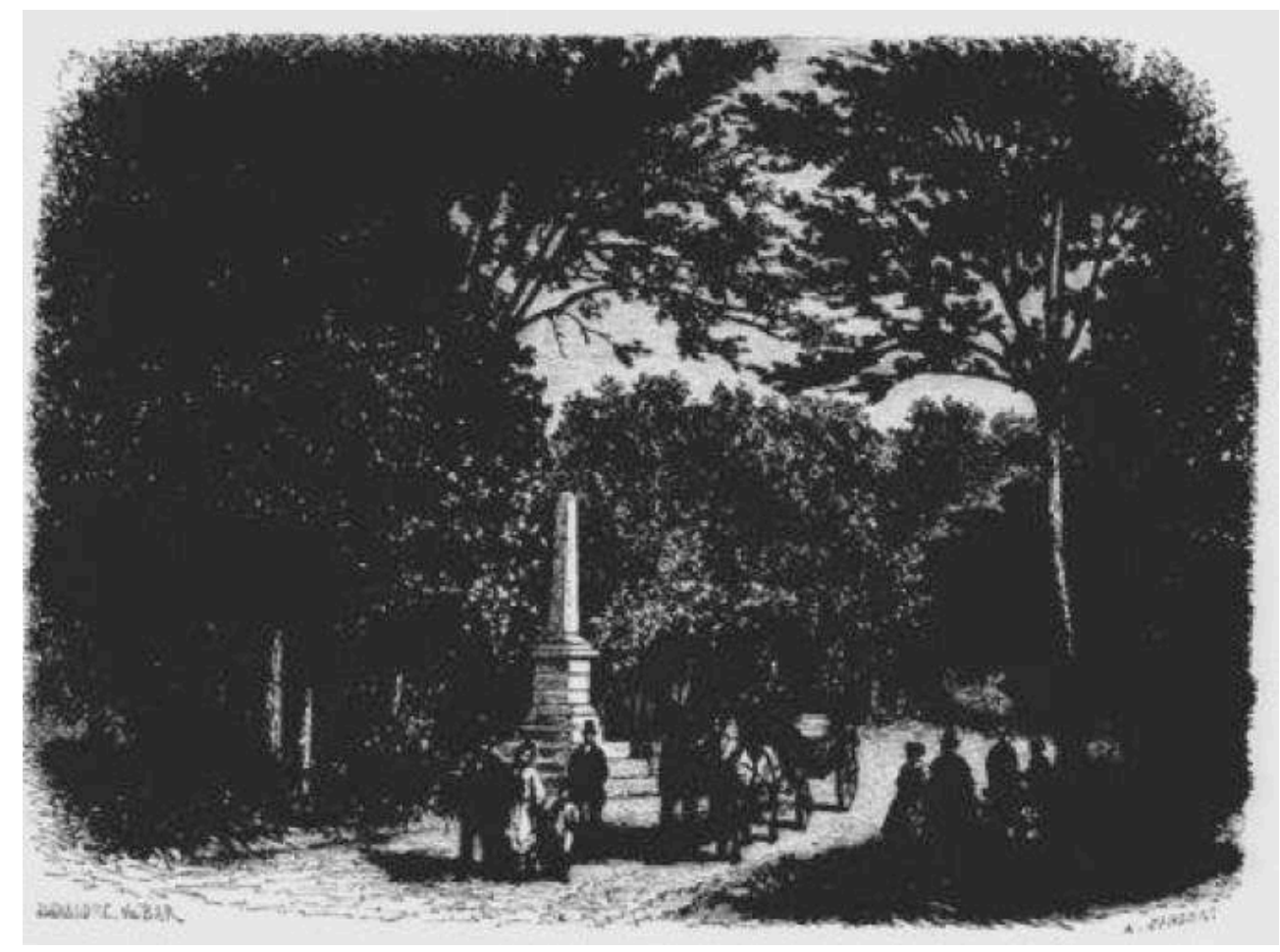

Pelouse de la Croix-Catelan. Source : Jean-Charles Adolphe Alphand, Les Promenades de Paris, «le Bois de Boulogne », op. cit. p. 51

Reste à savoir si Les Promenades de Paris ne sont pas représentatives d'une telle vision du loisir, qui servirait à souligner leur caractère normatif. Elles seraient le témoin d'un monde qui a effectivement changé. En effet, comme Chiara Santini le rappelle :

Remarquons aussi l'attention qui est portée à la description des usages et des pratiques des nouveaux jardins. Les paysages des squares et des différents aménagements sont presque toujours animés par des personnages. [...] C'est toute la bourgeoisie parisienne et ses pratiques de l'espace public que les Promenades mettent en scène. ${ }^{42}$ 
49 L'image qui suit a ceci de curieux qu'elle semble privatiser l'espace public, qui n'existerait que pour une seule famille ; car il est utopique d'imaginer un usage solitaire du Bois :

Fig. 7

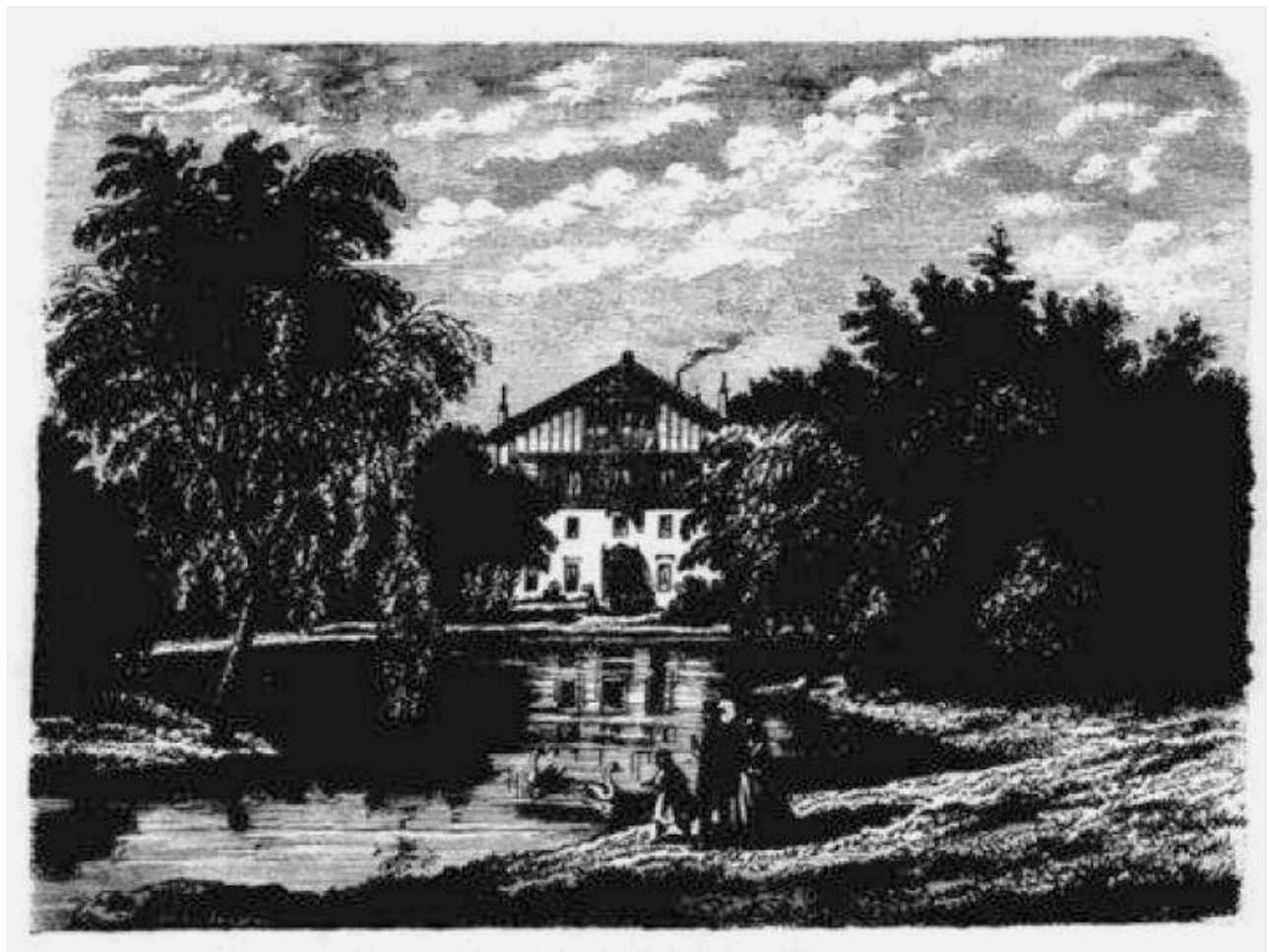

Pavillon d'Armenonville. Source : Jean-Charles Adolphe Alphand, Les Promenades de Paris, « le Bois de Boulogne », op. cit. p. 84

De la même manière, le Lac du Jardin d'Acclimatation semble bien vide, alors qu'il est une des attractions les plus populaires du bois de Boulogne : 
Fig. 8

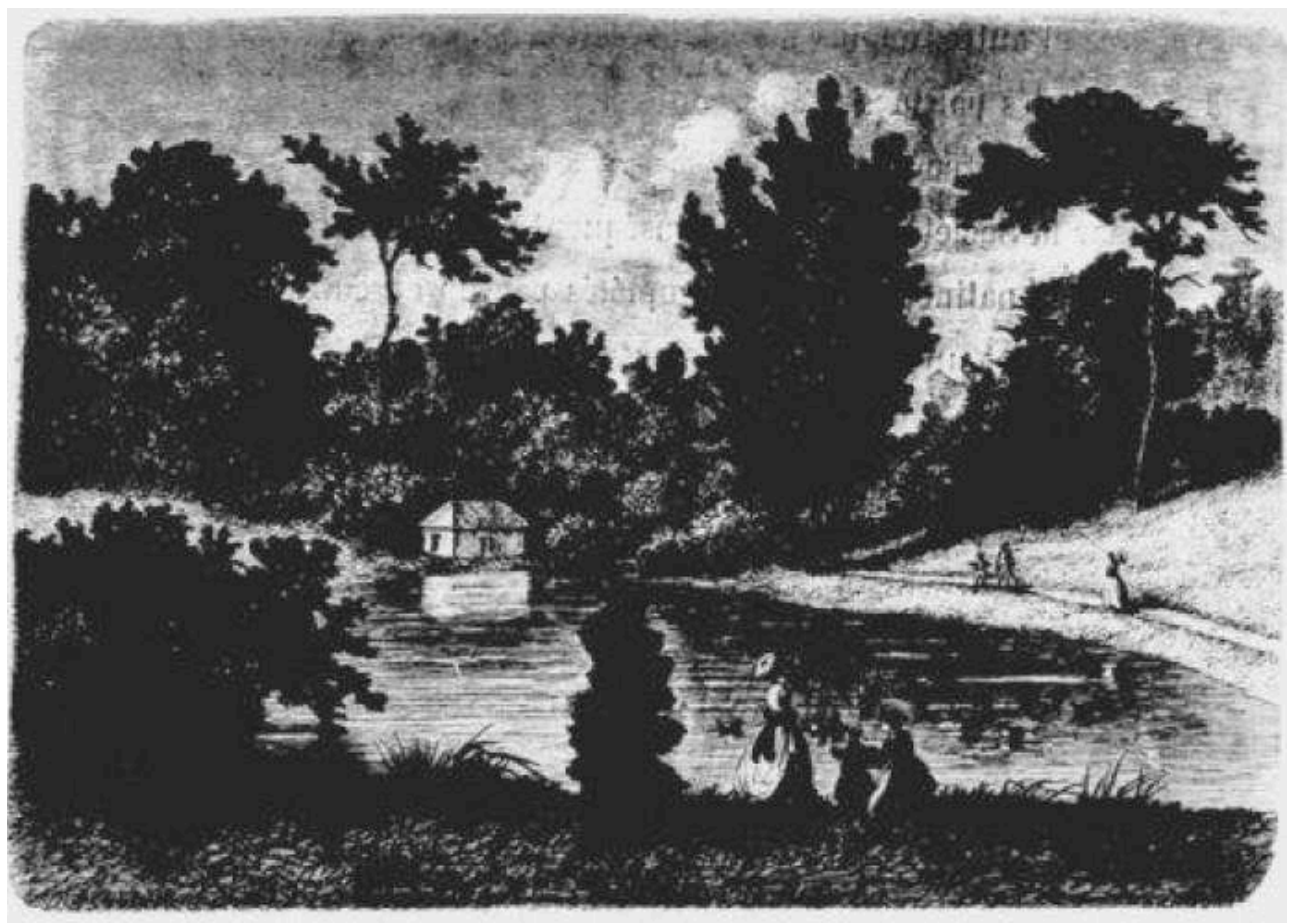

Lac du Jardin d'Acclimatation. Source : Jean-Charles Adolphe Alphand, Les Promenades de Paris, «le Bois de Boulogne», op. cit. p. 102

51 Il nous faut donc étudier la manière dont le livre prend en charge la diffusion d'un imaginaire qui n'est pas seulement la projection d'un homme (Alphand), ni d'un pouvoir (le Second Empire), mais bien celle d'une classe tout entière (la bourgeoisie). Pour cela, nous nous attarderons sur la représentation qu'offre Alphand du Bois de Boulogne. Parce qu'une figure essentielle de cet espace symbolique est absente de toutes ces illustrations : l'Empereur.

52 Une allusion d'Alphand au détour d'un bilan comptable, très évasive, nous étonne : alors qu'il compte « 102154 arbres, et 8428 bancs » à l'intérieur de Paris, il mentionne « les douloureux événements que Paris vient de traverser » pour préciser que «le tiers environ [en] a été détruit ou perdu $»^{43}$. L'auteur fait référence au Siège de Paris ainsi qu'à la Commune, qui marquent la chute de l'Empire. Le guide acte donc la fin d'une époque, dont il fut un témoin de la magnificence. Les Promenades de Paris, du fait de sa date de publication (1867-1873), serait le chant du cygne de l'urbanisme impérial, en même temps qu'il en acte la permanence. Il ne s'agit pas d'une simple adaptation au temps présent: un mode de vie s'est installé pendant la période impériale, qui a perduré sous la République jusqu'à l'entre-deux-guerres.

53 Le parcours d'Alphand a de quoi surprendre : fonctionnaire illustre de l'administration de Napoléon III, il maintient son rang au début de la IIIe République. Il remplace Haussmann, et parvient à se maintenir au plus haut niveau de la hiérarchie. Cela est étonnant, au premier abord: on se serait attendu à ce que la République nouvelle change d'état-major; bien au contraire. Il ne s'agit pas ici d'interroger le comportement d'Alphand durant cette période ${ }^{44}$ : à l'inverse du baron Haussmann, Alphand n'est pas ouvertement méprisant à l'égard des classes populaires. Il s'agit 
plutôt de comprendre à quoi correspond cette permanence à l'intérieur d'une transition politique exceptionnelle.

54 Le Bois de Boulogne était un espace de fête, consacré aux divertissements familiaux en même temps qu'à sa visibilité : un espace de consommation et d'admiration, typique d'une classe de loisir capable d'afficher ce que Veblen nomme un «loisir ostentatoire $»^{45}$, c'est-à-dire une activité s'affichant dans sa pleine inutilité et gratuité, caractéristique d'une certaine aristocratie. Comme l'écrit Julia Csergo :

En 1860, le Bois, remis à la mode par un souverain qui ne dédaigne pas d'y faire des apparitions régulières, s'agrémente du Jardin zoologique fondé par la Société impériale d'acclimatation [...] ; des écuries, une magnanerie, une grande volière, un aquarium [...] une serre aménagée en salon de lecture et en buffet offrent aux promenades, souvent familiales, une panoplie de divertissement gais, exotiques et inventifs autour de nouveaux commerces du loisir. À l'issue de ces transformations, le bois de Boulogne acquiert la réputation de «plus belle promenade de l'Europe. ${ }^{46}$

Napoléon III est l'instigateur d'un usage aristocratique du Bois de Boulogne, où les visiteurs sont capables de dépenser pour des choses inutiles, de payer pour du divertissement. Il marque durablement la pratique de la promenade, reprenant un usage hérité du XvIII ${ }^{e}$ siècle, typique d'une sociabilité de la distinction, qui est celui de la parade. Dans l'illustration qui suit, on perçoit un des véhicules privilégiés de cette parade aristocratique : la voiture, pour laquelle le Bois tout entier a été aménagé. Elle sépare ceux qui n'ont pas à parcourir le Bois de ceux qui y marchent et doivent se reposer sur les bancs. Il est significatif qu'elle soit l'espace dans lequel commence $L a$ Curée de Zola, roman de la haute-bourgeoisie et de l'aristocratie parisienne. On peut traverser le Bois de différentes manières, et les véhicules utilisés sont autant d'indices de distinction.

Fig. 9

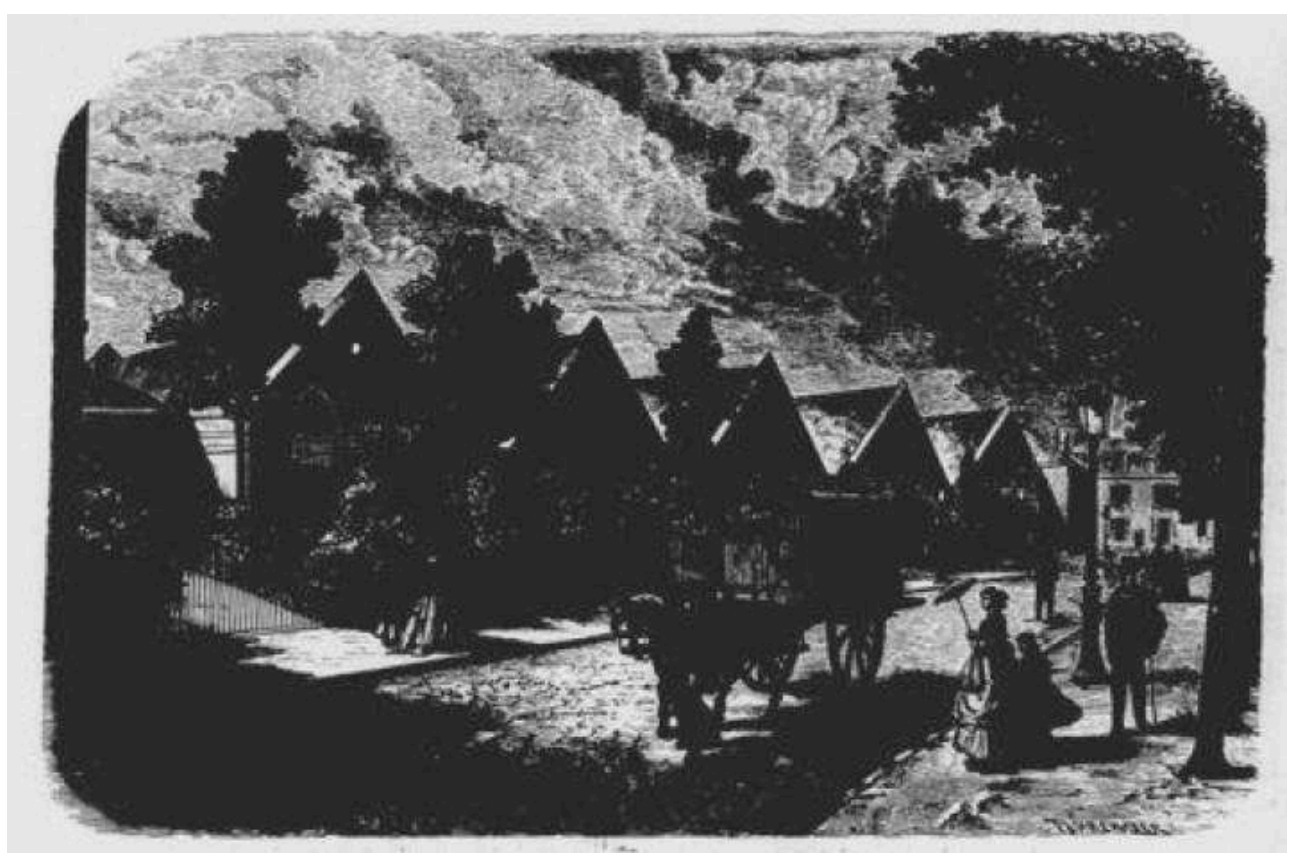

Extérieur des Glacières du bois de Boulogne. Source : Jean-Charles Adolphe Alphand, Les Promenades de Paris, "le Bois de Boulogne», op. cit. p. 111 

l'Ancien Régime disparaît. Avec l'amélioration technique, le progrès social se met en place. La démocratisation de l'espace public n'est effectivement qu'une chimère, dans l'usage des espaces verdoyants; cependant, la publicité qu'en fait leur principal organisateur montre que dans l'imaginaire collectif, cette démocratisation est effective. La disparition de l'Empire, de ses usages plutôt, était déjà actée avant sa chute, dans des pratiques qui attestaient d'une vision beaucoup plus «morale» de l'usage du loisir. Nulle contradiction, dès lors qu'Alphand puisse en poursuivre la publication sous la République: les espaces verts correspondent autant, sinon plus à l'idéologie républicaine qui en est à ses balbutiements. idéologie du loisir. Mais d'autres loisirs, bien plus dispendieux, formaient la sociabilité du bois de Boulogne. Il est significatif que plusieurs chapitres de La Curée, roman de l'avènement d'une nouvelle classe dominante sous le Second Empire, aient pour décor ce Bois, espace de la parade. Les femmes vont au Bois selon un cérémonial très développé, à partir de $15 \mathrm{~h}$, pour se montrer aux autres, et s'afficher dans leur puissance. C'est là un «loisir ostentatoire» puisque la promenade au Bois pour l'aristocratie impériale n'a pas d'autre objet qu'elle-même : celle de se montrer en train de se promener, de la même manière que l'Empereur se montre dans l'avenue de l'Impératrice pour affirmer symboliquement son autorité. Les illustrations des Promenades de Paris tendent à faire disparaitre ce type même d'ostentation: elles affichent un loisir plus discret, moins bruyant, et plus bourgeois qu'aristocratique.

En effet, les Promenades de Paris donnent à voir, dans diverses illustrations du Bois, la variété des activités où se projette une vie familiale idéale propre à la bourgeoisie. Partout on y voit des familles, dans une répartition harmonieuse de l'espace de la marche. Or, si ces images n'ont évidemment rien de faux, elles ne sont qu'une représentation partielle (et partiale) de la réalité. L'image du progrès vanté par Alphand est au service d'une idéologie de l'accès universel au loisir et au repos - celle du baron Haussmann qui l'a engagé. Comme le rappelle Emmanuel Pernoud, citant à plusieurs reprises les Mémoires du Préfet de la Seine :

Or cet homme [Haussmann] qui ne connaît ni le repos ni les dimanches va les organiser pour les autres. Cet homme pressé, infatigable, allergique aux «temps morts », va créer des espaces spécialement dévolus à la pause, aux moments creux, aux jours fériés. Décrits par Haussmann, les usagers du square sont « les petits, les humbles, les vaincus, les blessés de la lutte pour l'existence ${ }^{47}$

Les formules poétiques employées dans cette expression ne sont pas sans rappeler les descriptions des ouvriers dans les enquêtes de Villermé, où celui-ci mentionne la «perte de force », la «maigreur », le « teint flétri » des ouvriers de Mulhouse dans son Tableau de l'état physique et moral des ouvriers ${ }^{48}$. Pernoud poursuit son commentaire des Mémoires d'Haussmann, en mettant en avant le caractère médical de l'espace verdoyant, lieu de reconnaissance de la « lutte pour la vie » en son milieu :

Vu par Haussmann, le square n'est pas un lieu de plaisir mais de cure, la médication de ceux qui n'ont pas reçu de la nature les dispositions pour ne jamais s'arrêter, pour travailler tout le temps. [...] Le square s'occupe des corps : il restaure, il aère, il repose. Il n'existe pas sans cette préoccupation clinique autant que philanthropique des organismes fragiles ou fragilisés, ceux de l'enfant qui doit prendre des forces, de l'adulte qui doit les retrouver et du vieillard qui doit les ménager. Ses minces grilles tracent donc une frontière. Elles départagent l'humanité entre ceux qui travaillent sans fatigue les cinquante-deux dimanches de l'année et ceux qui n'en sont pas 
capables. La prose d'Haussmann traduit crûment le jugement sur la nature humaine qui présida à leur établissement. L'hygiénisme des squares ne trompe pas les observateurs qui rivalisent de termes cliniques pour les décrire. ${ }^{49}$

Comme son supérieur, le grand vœu d'Alphand est de reposer le promeneur. Par exemple, il explique comment les Buttes Chaumont ont été construites pour ne jamais fatiguer celui qui les gravit :

Des sentiers, dont les pentes n'excèdent pas 10 centimètres par mètre, mais exigent parfois des escaliers, donnent aux piétons la facilité de prendre des raccourcis entre les allées à voitures, et de s'élever jusqu'aux sommets du parc sans faire un trop long parcours..$^{50}$

61 Cela explique aussi pourquoi le Bois dispose de buffets et de restaurants, autant d'espaces où passer sa journée et se reposer. Le bois de Boulogne est pensé pour être un espace convivial autant qu'un espace de délassement. On voit dans les deux vignettes qui suivent que les restaurants et buffets ne sont pas les espaces d'un loisir "populaire », au sens péjoratif du terme. Ils sont au contraire des espaces où le calme rayonne, et d'où, encore, la foule est absente.

Fig. 10

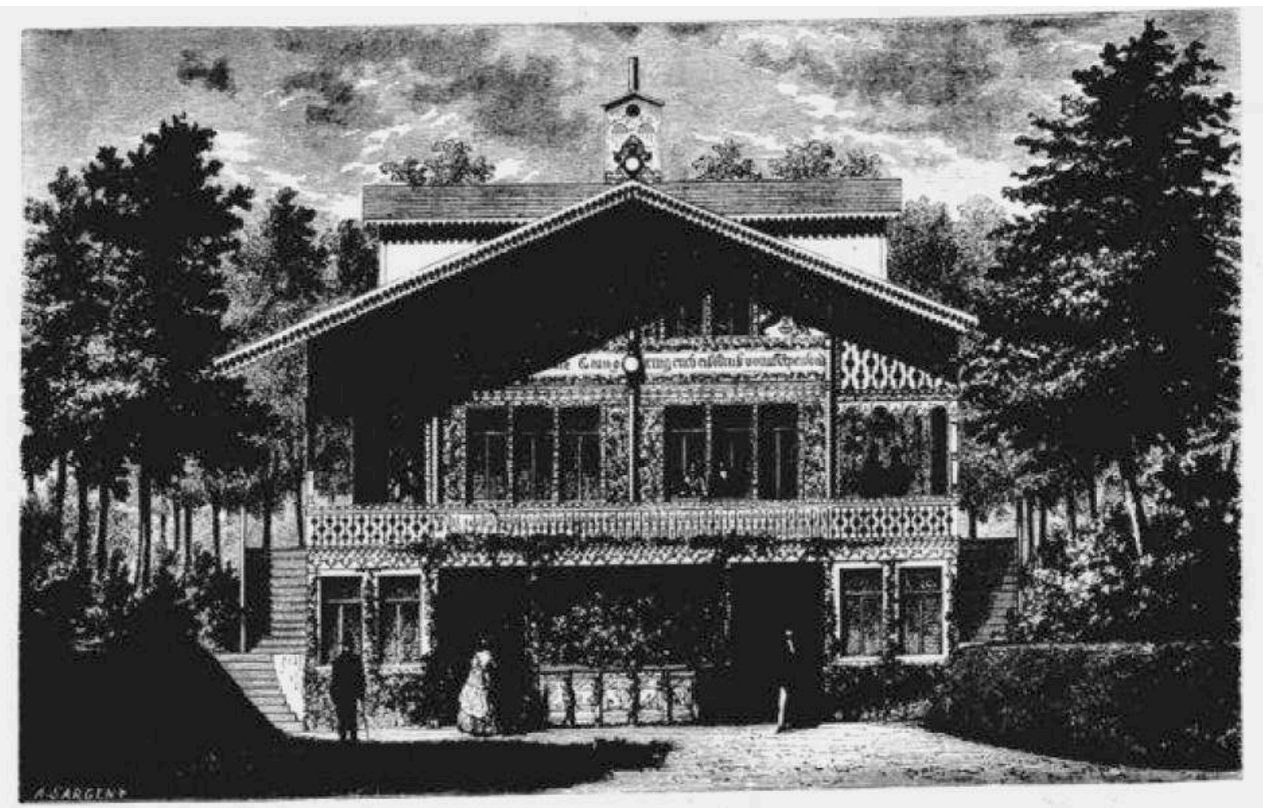

Restaurant de l'île. Source : Jean-Charles Adolphe Alphand, Les Promenades de Paris, «le Bois de Boulogne », op. cit. p. 81 
Fig. 11

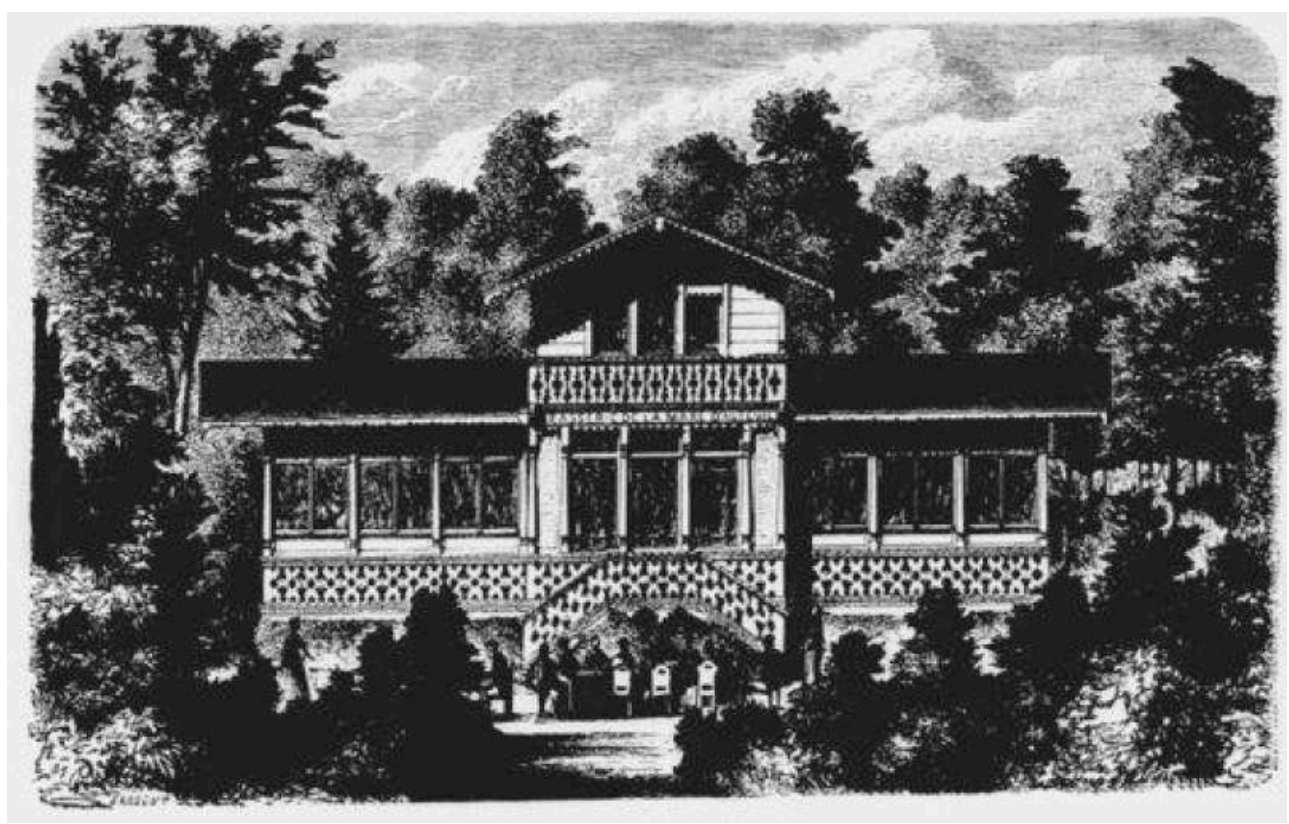

Buffet d'Auteuil. Source : Jean-Charles Adolphe Alphand, Les Promenades de Paris, «le Bois de Boulogne», op. cit. p. 87

Toutes les images que nous avons sélectionnées ${ }^{51}$, au cours de cette étude, sont surprenantes parce qu'elles révèlent de l'imaginaire des illustrateurs. Elles correspondent évidemment à une cible, et cherchent à attirer le public le plus large possible. N'oublions pas que les Promenades de Paris est un ouvrage de vulgarisation :

En complément d'articles élogieux, la presse reproduit aussi les gravures qui illustrent le livre. Ce procédé permet la circulation des images (et de l'imaginaire) des nouvelles promenades parisiennes auprès d'un public bien plus large que celui visé par l'ouvrage. ${ }^{52}$

Le but est de montrer que tous ces loisirs sont accessibles à chacun, en même temps que d'assurer leur singularité : les usagers des parcs trouveront un endroit privé, dans l'espace le plus fréquenté. Toutes ces images montrent des promenades familiales, de jour, et amoureuses, de nuit. À voir les illustrations, on est frappé par la constance d'un même schéma familial : la mère (ou nourrice) avec l'enfant. À chaque fois, on y imagine le calme et la douceur. On assiste à une exposition du mode de vie de la bourgeoisie. Par ces illustrations s'affirme une uniformisation de l'usage de l'espace public, qui ne semble jamais « public » paradoxalement, tant les scènes sont celles de la vie privée celles des jardins privatifs normalement. On a bien la preuve d'un usage normé de la ville dans Les Promenades de Paris.

On signalera l'effacement d'un autre élément courant du bois de Boulogne : la présence des prostituées. La raison de ce silence est évidente. Pourtant, le Bois est un haut-lieu de la prostitution, comme le rappelle Alain Corbin dans Les Filles de Noce qui mentionne le " grand nombre de «filles à parties" qui recrutent au Bois de Boulogne ou aux Champs Élysées $" .{ }^{53} \mathrm{Il}$ suffit de lire des nouvelles de Maupassant ${ }^{54}$ pour savoir à quoi correspond une "promenade au Bois ». L'imaginaire érotique qui lui est associé est immense : le tableau Promenade de Renoir, ou encore le poème "Dimanches Parisiens " d'Ephraïm Mickael suffisent à nous faire comprendre l'enjeu sexuel omniprésent dans 
ce lieu si propice au secret et à la cachette, quand Alphand en souligne la transparence et la constante visibilité :

[...]

Sur les promenades banales

Elles vont montrer leurs velours

Et les richesses hivernales

Des manteaux orgueilleux et lourds.

Elles passent, frêles poupées

Aux yeux cruellement sereins,

Adorablement occupées

À bien cambrer leurs souples reins,

A faire entrevoir leur chair d'ambre

Et leurs cheveux d'or blond ou roux,

Et, sur le verglas de Décembre,

Leur robe a de royaux froufrous.

Mais le long dimanche, plus triste

Que les plus monotones nuits,

Dans leurs yeux de froide améthyste

A mis la fièvre des ennuis.

$* *$

Ô Promeneuses des jours blêmes

D'hiver et des dimanches longs,

Nous, les chiffonneurs de poèmes,

Mignonnes, nous vous ressemblons [...]. ${ }^{55}$

Cet extrait du poème n'est qu'un exemple, éloquent, d'un usage bien différent du bois, illustré par la bohême. Le poème est intéressant, car il concentre tout ce qui caractérise le «dimanche » pour beaucoup de Parisiens, qui ne suivent pas l'usage bourgeois. Le dimanche est alors un temps d'inactivité, d'inertie: les dimanches sont «longs » et «tristes », les jours « blêmes » : les hypallages sont au service de l'expression lyrique du poète, dont le " Nous » de majesté renvoie à une catégorie de la société : les marginaux en proie à l'ennui. À Vincennes comme à Boulogne, Les Promenades de Paris s'en tiennent bien à l'écart, tentant de ne proposer dans Paris que des espaces clos, rassurants, promis à la sécurité. Jamais l'ouverture à un usage autre, dangereux, ne saurait être entendu pour des corps qui deviennent aussi policés que la nature qui les environne.

En effet, on est frappé par l'uniformité des corps dans toutes les illustrations : mêmes habits, mêmes postures, même loisirs. L'image qui suit ${ }^{56}$, d'un pavillon surveillant l'entrée, est finalement la marque même de ce succès de la bourgeoisie à l'intérieur de l'espace parisien : assurer une certaine autarcie des loisirs d'une classe, à horaires fixes, pour en faire un espace de pause, avec une utilité physique. En ce sens, on peut donc bien parler d'un usage prescriptif des Promenades de Paris. On nuancera cependant en signalant qu'il s'agit d'un usage implicite qui suit, plutôt qu'il n'impose, une technique réglée des corps modernes, qui informe l'imaginaire collectif. La teneur scientifique de ce discours est donc toute relative, Alphand étant, avant tout, un ingénieur et un homme de son temps, loin d'un Haussmann idéologue et « visionnaire ». 
Fig. 12

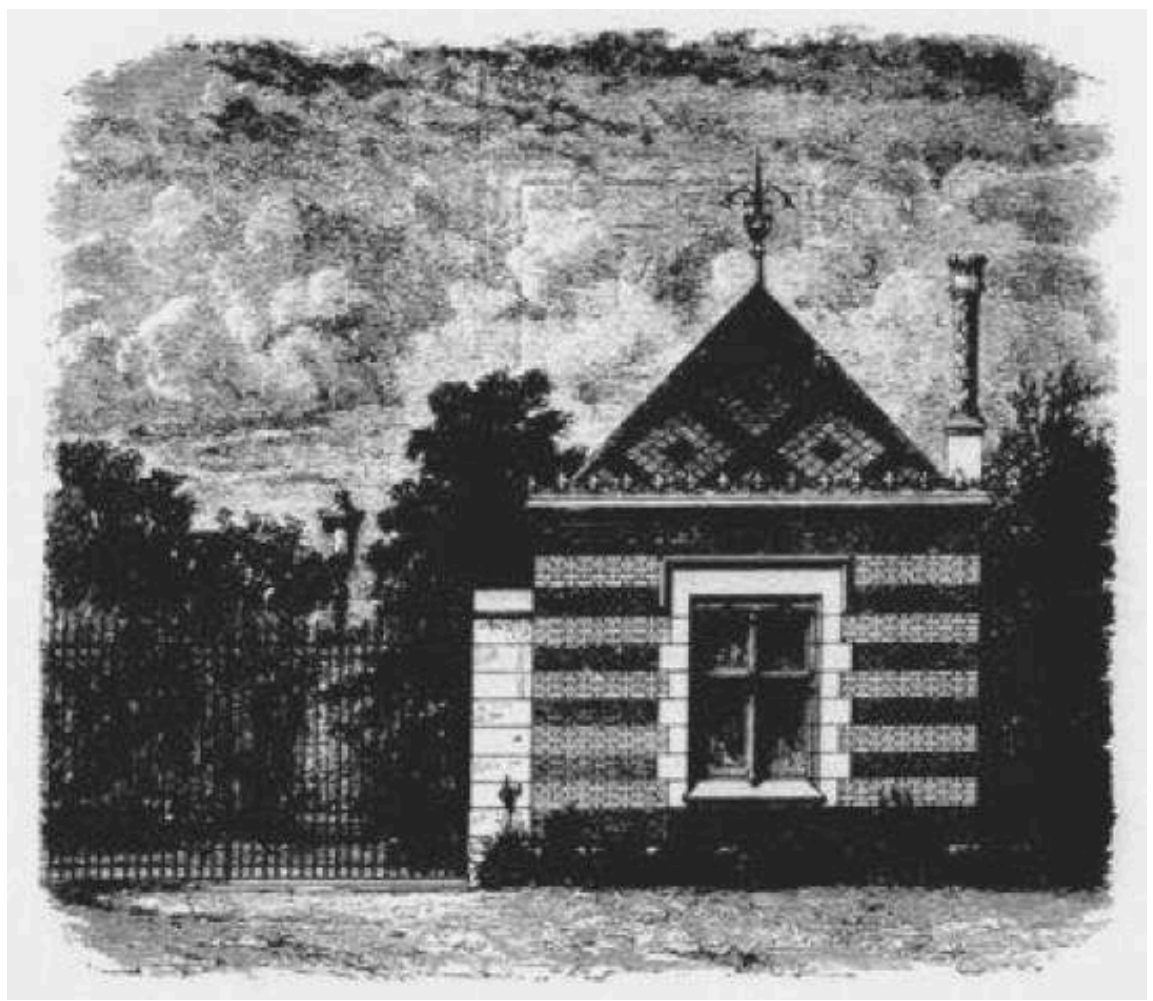

Porte Saint-James. Source : Jean-Charles Adolphe Alphand, Les Promenades de Paris, «le Bois de Boulogne», op. cit. p. 65

\title{
Conclusion : des espaces insensibles?
} vie contemplative à l'intérieur des grandes villes. Étonnamment, sa description n'est pas sans rappeler les illustrations analysées dans Les Promenades de Paris.

\begin{abstract}
$\$ 280$ - Architecture des hommes de connaissance. - Il faudra prendre conscience un jour, et vraisemblablement ce jour est-il proche, de ce qui manque avant tout à nos grandes villes: des lieux calmes et vastes, de vastes dimensions où méditer, des lieux possédant de longs portiques très spacieux pour le mauvais temps ou l'excès de soleil, où ne pénètre pas le vacarme des voitures et des bonimenteurs, et où une bienséance plus raffinée interdise même au prêtre de prier à voix haute: des édifices et des jardins qui expriment comme un tout la sublimité de la réflexion et $\mathrm{du}$ cheminement à l'écart. [...] Nous voulons nous être traduits en pierre et en plante, nous voulons nous promener en nous-mêmes, lorsque nous parcourons ces portiques (galeries) et ces jardins. ${ }^{57}$
\end{abstract}

Forme de renouvellement de la promenade rousseauiste, devenue mode d'existence et non plus soumission à un bannissement du monde, le texte de Nietzsche fait part d'un souhait, censé déjà exister depuis dix ans au moment où le livre est publié. Ultime preuve, peut-être, que l'ouvrage d'Alphand n'est que la fiction théorique d'un espace dont il offre la représentation fantasmée ; preuve, aussi d'un besoin important de repos qui caractérise la seconde moitié $\mathrm{du} \mathrm{XIX}^{\mathrm{e}}$ siècle, comme en témoignent les personnages de «surmenés » dans les romans naturalistes contemporains ${ }^{58}$. Ce repos nécessaire est 
la marque d'une accélération du monde, pour reprendre les analyses d'Hartmut Rosa dans son livre éponyme ${ }^{59}$.

Nous l'avons vu, les Promenades de Paris correspondent pleinement à l'avènement des loisirs, au sens où il est une publicité pour les futurs touristes parisiens, en même temps qu'il est la vitrine de l'usage des loisirs de la bourgeoisie de son temps. Seulement, le caractère idéalisé de cette représentation consacre aussi le risque de sa désillusion : à vendre un Paris rêvé, selon trois strates que sont le concepteur lui-même, le pouvoir qui l'instaure, et la catégorie sociale qui en a l'usage, il est probable que nombre de " consommateurs » de ce Paris soient déçus de l'écart entre la réalité et leur rêve.

Nous avons vu, en effet, que l'usage réel de nombre de ces parcs n'est pas celui vanté par Alphand. Ces espaces verts ne sont plus l'objet de plaisir, ni même l'objet de loisir : ils sont le lieu du repos. Ce repos nécessaire est perçu en même temps comme une réponse à un phénomène d'accélération du monde, dont les habitants des grandes villes sont les premières victimes. On en trouve la preuve dans l'instauration progressive d'un jour de repos pour l'ensemble d'une population. Comme l'écrit Julia Csergio :

Plutôt que jour de divertissement et de distraction, dimanche est jour de repos, de décélération, de vacance; hors de l'empreinte du travail, il renvoie aux temporalités physiologiques et sociales de l'homme et de la nature, au temps de l'épanouissement individuel et de la cohésion familiale. [...] Ainsi le dimanche est propre à un usage bucolique de la ville, à une régénération par la promenade et la marche au grand air, à un long instant de flânerie, dans un temps à soi, le long des boulevards, des parcs et des squares, dans les bois où les Parisiens les plus modestes pique-niquent et font jouer les enfants, dans un temps rythmé par le seul balancement de l'escarpolette ; en 1860 déjà, Jules Vallès relève l'attitude spécifique de cette foule tranquille et mélangée qui arpente les boulevards, le dimanche: "Ceux qui passent vont lentement et sans bruit. Ils se promènent. " $^{60}$

71 Vallès, dans sa description, montre une image négative de la promenade : celle-ci se fait « sans bruit », sans vie. Ce phénomène de la fatigue, de l'épuisement, que le personnage du médecin dans Charles Demailly relevait déjà, rejoint ce que Harmut Rosa a nommé une «crise de la résonance » dans son ouvrage éponyme ${ }^{61}$, ainsi que dans son court essai Rendre le monde indisponible ${ }^{62}$. Cette indisponibilité du monde se perçoit selon Rosa dans le développement de l'économie des loisirs, qui nous promet un repos que nous souhaitons, mais dont l'accès est bloqué par notre grande attente vis-à-vis de celui-ci. Nous cherchons tant à nous reposer, que nous devenons obsédés par cette idée, qui paradoxalement ne nous repose pas. La disponibilité économique du monde devient une indisponibilité sensible à celui-ci.

Les Promenades de Paris, en proposant de rendre un monde disponible au loisir, sont en fait la vitrine d'un monde qui ne l'est pas. Ce monde est en réalité réservé à une élite, et obéit déjà à une logique du service, de la consommation : le livre nous plonge dans la contradiction d'un loisir gratuit, qui est en fait un loisir normé. Toute personne, en y arrivant, constate l'impossibilité de pouvoir y participer : les héros de Maupassant, tel le père Leras de Promenade, en sont la preuve. Ces loisirs créent une distinction, et l'accès public à l'espace parisien est en fait une pseudo-démocratisation, où un mode de vie uniformisé s'instaure pour mieux séparer. Quand certains peuvent profiter du loisir, d'autres doivent se reposer: voilà aussi ce qu'illustre ce guide des promenades parisiennes. 


\section{NOTES}

1. Alain Corbin, Le Territoire du vide, l’Occident et le désir de rivage, chapitre v, A. « La généalogie des pratiques ", Paris, Aubier, 1988.

2. Pour davantage d'informations sur le parcours d'Adolphe Alphand, nous renvoyons aux travaux de Chiara Santini : " "De la science et de l'Art du paysage urbain" dans Les Promenades de Paris (1867-1873), traité de l'art des jardins publics ", dans Jean-Charles-Adolphe Alphand et le rayonnement des parcs publics de l'école française du XIXe siècle, Actes de la Journée d'Études du 22 mars 2017, organisée par l'École du Breuil, 2017, en ligne sur : https://www.culture.gouv.fr/Sitesthematiques/Monuments-Sites/Documentation/Actes-de-colloque/Actes-Journee-d-etude-2017Jean-Charles-Adolphe-Alphand-et-le-rayonnement-des-parcs-publics-de-l-ecole-francaise-duXIXe-siecle (consulté le 3 mai 2020) ; Id., "Construire le paysage de Paris. Alphand et ses équipes (1855-1891) », dans Michel Audouy, Jean-Pierre Le Dantec, Yann Nussaume, Chiara Santini (dir.), Le Grand Pari(s) d'Alphand. Création et transmission d'un paysage urbain, Paris, Éditions de La Villette, p. 32-49, 2018, p. 33) ; Id., Adolphe Alphand et la construction du paysage de Paris, Paris, Hermann, 2021.

3. Selon l'expression de Louis Chevalier, Classes laborieuses et classes dangereuses pendant la première moitié du XIXe siècle, Paris, Plon, 1958.

4. Selon l'expression de Thorstein Veblen, Théorie de la classe de loisir, traduction de Louis Évrard, préface de Raymond Aron, Paris, Gallimard, Collection « Tel », 1970.

5. Limido, Luisa, L'Art des jardins sous le Second Empire. Jean-Pierre Barillet-Deschamps,(1824-1873), Seyssel, Champ Vallon, 2002.

6. Si le détail des livraisons reste flou, nous pouvons dire que l'ouvrage était publié en 96 fascicules à partir de 1868, en deux grands volumes in-folio, et que les derniers fascicules étaient livrés à la fin de l'année 1872, avant une refonte éditoriale complète en 1873. Voir Chiara Santini, Adolphe Alphand et la construction du paysage de Paris, op. cit. chap. XI, «Théorie et Pratique de l'art des jardins publics ", «L'ingénieur-écrivain », p. 264

7. Alphand (dir.), Jean-Charles Adolphe, Les Promenades de Paris, (2 vol), Paris, J. Rothschild, 1867-1873, p. 53.

8. Alphand n'écrit que très peu, mais est un directeur d'ouvrage et un relecteur attentif. Voir Chiara Santini, Adolphe Alphand et la construction du paysage de Paris, op. cit. p. 266

9. Chiara Santini, «Promenades plantées et espaces verts: un regard historique sur la nature en ville de Paris ", Le Demeter, Paris, 2013, p. 211-226, p. 213.

10. Sur cette question de l'accès de l'art des jardins au domaine des Beaux-Arts, nous renvoyons à l'essai de Jacques Rancière, Le Temps du Paysage, III, «Le paysage comme la peinture », Paris, La Fabrique, 2020.

11. Louis-Michel Nourry, Les jardins publics en province. Espace et politique au XIXe siècle, Rennes, Presses Universitaires de Rennes, 1997.

12. Louisa Limido, Chiara Santini, "Raison et sentiment " du Paysage urbain, Adolphe Alphand et son équipe, conférence présentée au Musée de l'Arsenal, Paris, 25 novembre 2017, https:// www.youtube.com/watch ?v =4DyX2Kfgymk.

13. M. le Général Borgnis-Desbordes, président de l'Assemblée, Discours de l'Assemblée générale du 31 janvier 1892, Société Amicale de secours des anciens élèves de l'École polytechnique, Fonds Alphand, Paris, Bibliothèque de l'Hôtel de Ville.

14. Jules et Edmond Goncourt, cité dans Regards sur la peinture, «Le Lorrain ", n 49, p. 1, Paris, Éditions Fabbri, 1988.

15. Jacques Rancière, Le Temps du paysage, op. cit. p. 60. 
16. Jean-Charles Adolphe Alphand, Les Promenades de Paris, "Introduction ", "Choix du style à adopter dans la création d'un jardin » op. cit., p. 36.

17. Jean-Charles Adolphe Alphand, Les Promenades de Paris, « Le jardin romantique », op. cit., p. 47.

18. Ibid. p. 246.

19. Géraldine Texier-Rideau, «Le square haussmannien : l'espace public réinventé ", dans Simon Texier (dir.), Les Parcs et jardins dans l'urbanisme parisien: XIX $X^{e}-X^{e}$ siècles, Paris, AAVP, 2001, pp. 67-77, p. 71.

20. Alfred-Auguste (Baron) Ernouf, L'Art des jardins. Parcs - jardins - promenades. Étude historique principe de la composition des jardins - plantations. Décoration pittoresque et artistiques des parcs et jardins publics. Traité pratique et didactique par le baron Ernouf, troisième édition, entièrement refondue avec le concours d'Adolphe Alphand, "Le service des Promenades à Paris ", p. 347, Paris, J. Rothschild, 1886.

21. Jean-Charles Adolphe Alphand (dir.), Les Promenades de Paris, op. cit., p. 59 (nous soulignons).

22. Chiara Santini, «Promenades plantées et espaces verts », Art. cit. p. 215.

23. Jean-Charles Adolphe Alphand, Les Promenades de Paris, op. cit., p. 204.

24. Antoine Picon, «La pensée sociale et politique des ingénieurs des Ponts et Chaussées ", Pour mémoire, La Défense, Ministère de l'écologie du développement durable et de l'énergie Direction de la communication Département Images et édition, 2016.

25. Chiara Santini, "Construire le paysage de Paris. Alphand et ses équipes (1855-1891) ", dans Michel Audouy, Jean-Pierre Le Dantec, Yann Nussaume, Chiara Santini (dir.), Le Grand Pari(s) d'Alphand. Création et transmission d'un paysage urbain, Paris, Éditions de La Villette, 2018, p. 32-49.

26. Jean-Charles Adolphe Alphand (dir.), Les Promenades de Paris, op. cit., p. 59.

27. Jean-Baptiste Godin, Solutions Sociales, Paris, La Digitale, 1979 [1871].

28. Jessica DOS SANTOS, «Le Familistère de Guise: habitat collectif et autonomie ouvrière ", Revue du Nord, vol. 374, $\mathrm{n}^{\circ}$ 1, 2008, p. 63-76, ici p. 64-65.

29. Géraldine Texier-Rideau, Art. cit. p. 74

30. Emmanuel Pernoud, Paradis Ordinaires, l'artiste au jardin public, Paris, Les Presses du Réel, 2013, p. 84. Pour la citation de Jules Vallès, voir le Tableau de Paris, éd. de Maxime Jourdan, Paris, Berg, 2007, p. 294.

31. Chiara Santini, «Promenades plantées et espaces verts », Art. cit., p. 218.

32. Écrire le «Bois ", avec une majuscule, fait systématiquement référence au bois de Boulogne, au XIX ${ }^{\mathrm{e}}$ siècle.

33. Julia Csergo, "Extension et mutation du loisir citadin. Paris $\mathrm{XIX}^{\mathrm{e}}$-début $\mathrm{xx}$ siècle ", dans Alain Corbin (dir.), L'Avènement des Loisirs, Paris, Aubier, 1995, p. 119-168, ici p. 140.

34. Jean-Charles Adolphe Alphand, op. cit. p. 172.

35. Ibid., p. 58

36. Ibid., p. 159 (nous soulignons).

37. Ibid., p. 164.

38. Emmanuel Pernoud, Paradis Ordinaires, op. cit. p. 149.

39. Pour une analyse du phénomène de la Bohême, nous renvoyons aux analyses d'Alain Vaillant dans Charle, Christophe, Jeanpierre, Laurent, (dir.) La Vie intellectuelle en France, des lendemains de la Révolution à 1914, Paris, Le Seuil, 2016.

40. Edmond et Jules Goncourt, éd. Jean-Didier Wagneur et Françoise Cestor, Charles Demailly $\{1860\}$, chap. LVIII, Euvres narratives complètes, Tome III, Paris, Classiques Garnier, 2014, p. $429-430$. 
41. Edmond et Jules Goncourt, Journal des Goncourt, Mémoires de la vie littéraire, 18 janvier 1857, Tome premier : 1851-1861, p. 163-222, Paris, Bibliothèque-Charpentier, 1891.

42. Chiara Santini, « "De la science et de l'Art du paysage urbain” dans Les Promenades de Paris (1867-1873), traité de l'art des jardins publics», p.9-10, dans Jean-Charles Adolphe Alphand et le rayonnement des parcs publics de l'école française, Actes de la Journée d'Études organisée par la Direction générale des patrimoines et l'École du Breuil, 22 mars 2017.

43. Jean-Charles Adolphe Alphand, op. cit., p. 246.

44. Plusieurs anecdotes montrent le soutien indéfectible d'Alphand à chaque membre de ses équipes, à tous les échelons. De la même façon, la querelle de la Tour Eiffel montre sa capacité de dialogue et d'écoute, et les recensions de sa mort, dans la presse, sont assez unanimes à saluer les qualités humaines de l'individu.

45. Veblen, Théorie de la classe de loisir, « le loisir ostentatoire », op. cit.

46. Julia Csergo, Art. cit., p. 140.

47. Emmanuel Pernoud, Paradis ordinaires, op. cit. p. 24.

48. Louis-René Villermé, Tableau de l'état physique et moral des ouvriers employés dans les manufactures de coton, de laine et de soie, t. I, Paris, 1840, p. 30.

49. Emmanuel Pernoud, Paradis ordinaires, op. cit. p. 24.

50. Jean-Charles Adolphe Alphand, op. cit., p. 204.

51. Jean-Charles Adolphe Alphand, op. cit., fig. 32, p. 38; fig. 63, p. 51; fig. 68, p. 55; fig. 95, p. 81; fig. 100, p. 84; fig. 106, p. 87; fig. 120, p. 102; fig. 134, p. 111.

52. Chiara Santini, "De la science et de l'Art du paysage urbain" dans Les Promenades de Paris (1867-1873), traité de l'art des jardins publics ", Art. cit., p. 11.

53. Alain Corbin, Les Filles de Noce : misère sexuelle et prostitution aux XIX et XX $X^{e}$ siècles, Paris, Champs Flammarion, 1982 [1978], p. 131.

54. Par exemple, Promenade, qui relate le suicide d'un fonctionnaire désillusionné après une promenade au Bois qui d'enchanteresse devient le moment d'une crise existentielle, après la rencontre d'une prostituée. Voir Maupassant, «Promenade », Gil Blas du 27 mai 1884, https:// athena.unige.ch/athena/maupassant/maupassant-promenade.html/

55. Ephraïm Mikhael, CEuvres, Poésies, Paris, éd. d'A. Lemerre, 1890.

56. Jean-Charles Adolphe Alphand, op. cit., p. 65

57. Friedrich Nietzsche, Le Gai Savoir, \$280, trad. De Patrick Wotling, Paris, GF-Flammarion, 2007 [1997], p. 229.

58. Que l'on songe chez Zola au Ventre de Paris, au Bonheur des Dames, à La Joie de vivre, à L'Euvre, ou chez les Goncourt à Renée Mauperin, ou à Charles Demailly, qui présentent tous des personnages épuisés par la ville, et l'accélération du monde.

59. Hartmut Rosa, Accélération, une critique sociale du temps, trad. de Didier Renault, Paris, La Découverte, 2010.

60. Julia Csergo, Art. cit. p. 167

61. Hartmut Rosa, Résonance, une sociologie de la relation au monde, trad. De Sacha Zilberfarb \& Sarah Raquillet, Paris, La Découverte, 2018.

62. Hartmut Rosa, Rendre le monde indisponible, trad. D’Olivier Mannoni, Paris, La Découverte, 2020. 


\section{RÉSUMÉS}

Adolphe Alphand a été responsable du département des Promenades et Plantations de la ville de Paris, de 1855 à 1891. Des travaux monumentaux de Paris, l'ingénieur a tiré un livre, Les Promenades de Paris. Si l'apologie y est évidente, d'autres points sont à relever. Participant pleinement à l'idéologie hygiéniste de son siècle, Les Promenades de Paris marque aussi un regard nouveau sur la pratique même de la promenade: de ses usages, finalités, et enjeux. Derrière l'aspect strictement descriptif d'un kaléidoscope des promenades dans Paris et ses Bois, nous interrogerons donc le caractère prescriptif de l'ouvrage.

Adolphe Alphand was manager of the "Promenades and Plantations" Department in Paris, from 1855 to 1891 . He edited a book from those monumental public works, Les Promenades de Paris. The apologetic aspect of this book is obvious. Nevertheless, other points have to be mentioned: more than involved in the hygienist ideology of the century, Les Promenades de Paris is also a new vision of the practice of walking. Behind a descriptive aspect of those walks inside of Paris, we will study the prescriptive aspect of Les Promenades de Paris.

INDEX

Mots-clés : promenades, Alphand, norme de vie, bourgeoisie, loisirs

\section{AUTEUR}

\section{ÉDOUARD BOURDELLE}

Université de Strasbourg (ED520). 